\title{
New constraints on the chemical evolution of the dwarf spheroidal galaxy Leo I from VLT spectroscopy ${ }^{\star}$
}

\author{
M. Gullieuszik ${ }^{1}$, E. V. Held ${ }^{1}$, I. Saviane ${ }^{2}$, and L. Rizzi ${ }^{3}$ \\ 1 Osservatorio Astronomico di Padova, INAF, vicolo dell'Osservatorio 5, 35122 Padova, Italy \\ e-mail: [marco.gullieuszik; enrico.held]@oapd.inaf.it \\ 2 European Southern Observatory, Casilla 19001, Santiago 19, Chile \\ e-mail: isaviane@eso.org \\ 3 Joint Astronomy Centre, 660 N. A’ohoku Place, University Park, Hilo, HI 96720, USA \\ e-mail: 1.rizzi@jach.hawaii.edu
}

Received 23 December 2008 / Accepted 27 March 2009

\begin{abstract}
We present the spectroscopy of red giant stars in the dwarf spheroidal galaxy Leo I, aimed at further constraining its chemical enrichment history. Intermediate-resolution spectroscopy in the Ca II triplet spectral region was obtained for 54 stars in Leo I using FORS2 at the ESO Very Large Telescope. The equivalent widths of Ca II triplet lines were used to derive the metallicities of the target stars on the $[\mathrm{Fe} / \mathrm{H}]$ scale of Carretta \& Gratton, as well as on a scale tied to the global metal abundance, $[\mathrm{M} / \mathrm{H}]$. The metallicity distribution function for red giant branch (RGB) stars in Leo I is confirmed to be very narrow, with mean value $[\mathrm{M} / \mathrm{H}] \simeq-1.2$ and dispersion $\sigma_{[\mathrm{M} / \mathrm{H}]} \simeq 0.2$. By evaluating all contributions to the measurement error, we provide a constraint to the intrinsic metallicity dispersion, $\sigma_{[\mathrm{M} / \mathrm{H}], 0}=0.08$. We find a few metal-poor stars (whose metallicity values depend on the adopted extrapolation of the existing calibrations), but in no case are stars more metal-poor than $[\mathrm{Fe} / \mathrm{H}]=-2.6$. Our measurements provide a hint of a shallow metallicity gradient of -0.27 dex $\mathrm{Kpc}^{-1}$ among Leo I red giants. The gradient disappears if our data are combined with previous spectroscopic datasets in the literature, so that any firm conclusions about its presence must await new data, particularly in the outer regions. By combining the metallicities of the target stars with their photometric data, we provide age estimates and an age-metallicity relation for a subset of red giant stars in Leo I. Our age estimates indicate a rapid initial enrichment, a slowly rising metal abundance - consistent with the narrowness of the metallicity distribution - and an increase of $\sim 0.2$ dex in the last few Gyr. The estimated ages also suggest a radial age gradient in the RGB stellar populations, which agrees with the conclusions of a parallel study of asymptotic giant branch stars in Leo I from near-infrared photometry. Together, these studies provide the first evidence of stellar population gradients in Leo I.
\end{abstract}

Key words. galaxies: dwarf - galaxies: individual: Leo I - stars: abundances - Local Group - galaxies: stellar content

\section{Introduction}

A first-order estimate of the distribution of stellar metallicities in resolved galaxies can be obtained from photometry of red giant branch (RGB) stars. However, young metal-rich stars have the same colours as older metal-poor stars, a phenomenon known as "age-metallicity degeneracy". Although improved photometric metallicity estimates can be obtained by combining optical and near-infrared photometry, with reduced degeneracy effects (Gullieuszik et al. 2007, 2008), the problem is never entirely overcome, and age and metallicity inextricably contribute to the observed colour.

More direct measurements of the stellar metallicity distribution function (MDF) are obtained from spectroscopy. The most accurate determinations come from high-resolution abundance analysis which provides information on the relative abundances of chemical elements, and therefore the strongest constraints to the galaxy evolution models (e.g. Tolstoy et al. 2003; Lanfranchi \& Matteucci 2007). High-resolution spectroscopy, however, is limited to the brightest stars in nearby dwarf galaxies, and becomes unfeasible for more distant galaxies even within the Local Group and using $10 \mathrm{~m}$-class telescopes. A viable

* Based on data collected at the European Southern Observatory, Paranal, Chile, Proposals No. 69.D-0455 and 71.D-0219. alternative for obtaining metallicities for a large number of stars (as needed to derive statistically significant stellar MDFs) is using low- or intermediate-resolution spectroscopy. The infrared Ca triplet (CaT) method, originally devised to measure metallicities of stars in Galactic globular clusters (Armandroff \& Zinn 1988; Armandroff \& Da Costa 1991; Rutledge et al. 1997b), has now been applied quite extensively to RGB stars in dwarf galaxies. Using this method, values of $[\mathrm{Fe} / \mathrm{H}]$ for dwarf galaxies have been obtained for Fornax (Tolstoy et al. 2001; Pont et al. 2004; Battaglia et al. 2006), Carina (Koch et al. 2006), Sculptor (Tolstoy et al. 2001), Leo II (Bosler et al. 2007; Koch et al. 2007a), NGC 6822 (Tolstoy et al. 2001) and the LMC (Cole et al. 2005).

In this paper we present new CaT spectroscopy for the dwarf spheroidal (dSph) galaxy Leo I. Leo I is known to have formed the bulk of its stars at an intermediate epoch (e.g., Gallart et al. 1999). Along with Leo II, Leo I is one the most distant dSph satellites of the Milky Way, for which the influence of tidal interaction with our Galaxy on evolution must have been more limited than for nearby galaxies such as Carina or Fornax. As such, deriving its chemical enrichment history is of foremost importance for our knowledge of the evolution of dSph galaxies. Gallart et al. (1999) derived the metallicity and reconstructed the star-formation history (SFH) of Leo I from HST/WFPC2 data - they found a metallicity ranging from 
$[\mathrm{Fe} / \mathrm{H}]=-1.4$ to $[\mathrm{Fe} / \mathrm{H}]=-2.3$. Using the same data set, Dolphin (2002) found higher metallicity, ranging from $[\mathrm{Fe} / \mathrm{H}]=$ -0.8 to $[\mathrm{Fe} / \mathrm{H}]=-1.2$. From the colour of RGB stars, Held et al. (2000) derived a mean metallicity $[\mathrm{Fe} / \mathrm{H}] \sim-1.6$ on the Zinn \& West (1984) scale.

The metallicity distribution of RGB stars in Leo I has recently been investigated by Bosler et al. (2007) and Koch et al. (2007b). Bosler et al. (2007) used the CaT method to analyse Keck-LRIS spectra of 102 RGB stars, and found $[\mathrm{Fe} / \mathrm{H}]=$ -1.34 on the $[\mathrm{Fe} / \mathrm{H}]$ scale of Carretta \& Gratton $(1997$, hereafter, CG97). The authors also proposed a new calibration based on $\mathrm{Ca}$ abundance, yielding a mean metallicity $[\mathrm{Ca} / \mathrm{H}]=-1.34$ $(\sigma=0.21)$. Also using measurements of the Ca II triplet lines, Koch et al. (2007b) found a mean metallicity $[\mathrm{Fe} / \mathrm{H}]=-1.31$ on the CG97 scale for 58 red giants. In both studies, the MDF is well described by a Gaussian function with a $1 \sigma$ width of $0.25 \mathrm{dex}$, and a full range in $[\mathrm{Fe} / \mathrm{H}]$ of approximately $1 \mathrm{dex}$.

Leo I is an interesting target also because, to date, it is one of the few Local Group dwarf galaxies showing scarce evidence of a population gradient. Held et al. (2000) found that the old horizontal branch stars of Leo I are radially distributed as the intermediate-age helium-burning stars; Koch et al. (2007b) found no significant metallicity radial gradient. Different conclusions were found in our companion paper, based on near-infrared photometry (Held et al. 2009), showing that intermediate-age asymptotic giant branch (AGB) stars are more concentrated in the central region than old RGB stars.

A new, independent data set of metallicities for Leo I stars, also based on the CaT method, was obtained by us at the ESO VLT using high signal-to-noise FORS2 spectra. The new sample has negligible overlap with the previous data sets, thus effectively increasing the number of Leo I stars with direct metallicity measurements. Using the new data, this paper provides an independent determination of the MDF of Leo I and further constraints on its evolution, based on an analysis of metallicity and age gradients and the age-metallicity relation.

\section{Observations and reduction}

\subsection{Target selection}

Our targets were selected from the colour-magnitude diagram (CMD) of Leo I. For the central region of the galaxy, we relied upon $B, V$-band photometry from Held et al. (2000), obtained with the EMMI instrument at the NTT telescope at the ESO La Silla Observatory. For the stars in the outer regions, we used the $B V$ photometry originally obtained for a study of RR Lyrae variable stars in Leo I (Held et al. 2001), based on observations carried out with the Wide Field Imager at the $2.2 \mathrm{~m}$ ESO-MPI telescope.

The spectroscopic targets were selected among the brightest RGB stars of Leo I, down to 1 mag below the RGB tip. We avoided any colour constraints that might bias the age/metallicity distribution. After mask design (for which we were guided only by geometric constraints) we were left with 61 targets in 4 masks.

The identifiers, coordinates, and $B V$ photometry of the stars in our final sample (excluding a few targets with too low $S / N$ ratio to allow any measurements) are listed in Table 1 , and the targets are shown in Fig. 1. The location of the target stars in the CMD of Leo I is shown in Fig. 2.

\subsection{Observations}

The observations were carried out in service mode in two runs between May 2002 and December 2003 using FORS2, the multi-mode optical instrument mounted on the Cassegrain focus of the Yepun (VLT-UT4) $8.2 \mathrm{~m}$ telescope at the ESO Paranal Observatory. We used FORS2 in MXU mode with the $1028 z+29$ grism and the OG590+32 order-blocking filter. With this setup, the spectral coverage is approximately $7700 \AA$ to $9500 \AA$, with a dispersion $0.85 \AA$ pixel $^{-1}$. The selected targets were observed with 4 masks using 0.'80 slits. For each Leo I mask, two spectra were taken. The observing log and exposure times are given in Table 2. We observed also RGB stars in 8 Galactic globular clusters (GCs) in a wide range of metallicity with the same instrumental setup in order to calibrate our measurements onto a known metallicity scale. Two different exposure times were used (long and short exposure) to prevent saturation of the brightest RGB stars. The photometry of the GC stars was taken from the data compilation of Rutledge et al. (1997b).

\subsection{Data reduction}

The basic reduction of multi-object spectra was performed using standard procedures in IRAF $^{1}$. Bias and flat-field corrections were applied to all images using the ccdproc task. Due to the long exposure times, the scientific frames for Leo I contain a large number of cosmic ray hits. These were effectively cleaned using the IRAF program lacos (van Dokkum 2001) on the bias-subtracted, flat-fielded images. The multi-object spectra were extracted with the apall task and wavelength calibrated using HeNeAr lamp exposures taken at the end of each night.

The two spectra taken for each Leo I target were combined to increase the $S / N$ ratio. However, we also retained the two individual spectra to estimate the uncertainties in the wavelength calibration and line strengths. Finally, the continuum was normalised in the region between $8400 \AA$ and $8800 \AA$, by excluding in the process the $\mathrm{Ca}$ II and other relatively strong absorption lines. Typical sky-subtracted spectra of three Leo I stars with different metallicities are shown in Fig. 3.

The average signal-to-noise ratio per pixel was calculated from the rms of the combined spectra in two wavelength windows free from strong spectral features, 8580-8620 ̊ and 8710-8750 ̊. The values were checked against those measured on the raw spectra, and found to be consistent within a few percent. The $S / N$ ratio, listed in Table 1 , is $\gtrsim 20$ for all stars in our sample, with a mean value of $\sim 50$.

\section{Radial velocities and membership}

Radial velocities were measured for target stars to establish their membership. Only the two reddest lines were used to this purpose, since the bluest line of the $\mathrm{Ca}$ triplet is weak and, for the systemic velocity of LeoI, overlapping with a strong sky line. The line wavelengths were obtained from the central values $\lambda_{m}$ of the fitted profile (see Eq. (1) below), and compared with the laboratory air wavelengths $8542.09 \AA$ and $8662.14 \AA$. A radial velocity was measured for each individual spectrum of each star by combining the measurements of the two lines $\lambda_{8542}$ and $\lambda_{8662}$. Then, the radial velocity was calculated as the mean of

\footnotetext{
1 IRAF is distributed by the National Optical Astronomy Observatory, which is operated by the Association of Universities for Research in Astronomy (AURA) under cooperative agreement with the National Science Foundation.
} 
Table 1. Spectroscopic sample in the Leo I field.

\begin{tabular}{|c|c|c|c|c|c|c|c|c|}
\hline ID & $\bar{~} \overline{\alpha(\mathrm{J} 2000)}$ & $\delta(\mathrm{J} 2000)$ & $B-V$ & $V$ & $\begin{array}{c}v \\
\mathrm{~km} \mathrm{~s}^{-1}\end{array}$ & $\begin{array}{c}\Delta v \\
\mathrm{~km} \mathrm{~s}^{-1}\end{array}$ & $S / N$ & Other \\
\hline 1 & $10: 08: 28.43$ & $+12: 15: 54.1$ & 1.39 & 19.62 & 268.2 & 1.5 & 57 & \\
\hline 2 & 10:08:16.47 & $+12: 15: 59.5$ & 1.48 & 19.59 & 264.5 & 3.2 & 46 & \\
\hline 3 & $10: 08: 36.41$ & $+12: 16: 02.2$ & 1.46 & 19.89 & 261.9 & 5.7 & 37 & \\
\hline 4 & $10: 08: 17.58$ & $+12: 16: 15.7$ & 1.61 & 19.55 & 267.0 & 0.9 & 42 & \\
\hline 5 & 10:08:19.45 & $+12: 16: 34.1$ & 1.10 & 20.28 & 268.6 & 3.2 & 32 & B8391 \\
\hline 6 & 10:08:40.46 & $+12: 16: 38.0$ & 1.35 & 19.78 & 273.5 & 0.4 & 36 & B25113 \\
\hline 7 & $10: 08: 31.00$ & $+12: 16: 40.6$ & 1.32 & 19.85 & 282.4 & 3.1 & 58 & B18214 \\
\hline 8 & 10:08:03.62 & $+12: 16: 46.4$ & 1.50 & 19.74 & 301.0 & 7.4 & 59 & \\
\hline 9 & $10: 08: 32.56$ & $+12: 16: 54.9$ & 1.19 & 20.45 & 290.7 & 1.5 & 33 & \\
\hline 10 & 10:08:08.79 & $+12: 17: 05.9$ & 1.07 & 20.00 & 304.3 & 2.2 & 90 & \\
\hline 11 & 10:08:10.61 & $+12: 17: 08.0$ & 1.20 & 20.05 & 262.2 & 6.8 & 62 & B4173 \\
\hline 12 & $10: 08: 49.42$ & $+12: 17: 14.8$ & 1.18 & 20.44 & 281.2 & 2.5 & 18 & \\
\hline 13 & $10: 08: 34.90$ & $+12: 17: 17.8$ & 1.16 & 20.01 & 287.8 & 6.8 & 38 & \\
\hline 14 & 10:08:39.66 & $+12: 17: 19.9$ & 1.37 & 19.68 & 258.4 & 3.7 & 44 & K195 \\
\hline 15 & 10:08:57.38 & $+12: 17: 20.8$ & 1.18 & 20.44 & 280.3 & 6.6 & 34 & K833 \\
\hline 16 & 10:08:55.47 & $+12: 17: 21.9$ & 1.26 & 20.14 & 274.2 & 10.9 & 43 & \\
\hline 17 & 10:08:11.82 & $+12: 17: 29.4$ & 1.05 & 20.31 & 260.0 & 0.6 & 51 & \\
\hline 18 & 10:08:29.89 & $+12: 17: 31.7$ & 1.30 & 19.67 & 262.8 & 4.8 & 43 & \\
\hline 19 & 10:08:07.52 & $+12: 17: 34.6$ & 1.25 & 20.16 & 269.6 & 7.4 & 52 & \\
\hline 20 & 10:08:14.21 & $+12: 17: 36.1$ & 1.11 & 19.90 & 247.6 & 32.2 & 67 & B5496 \\
\hline 21 & 10:08:15.40 & $+12: 17: 38.3$ & 1.43 & 19.48 & 266.1 & 3.0 & 37 & \\
\hline 22 & 10:08:46.36 & $+12: 17: 41.3$ & 1.33 & 20.32 & 261.9 & 2.9 & 30 & \\
\hline 23 & 10:08:20.50 & $+12: 17: 45.0$ & 1.61 & 19.64 & 263.7 & 0.3 & 50 & \\
\hline 24 & 10:08:06.29 & $+12: 17: 45.3$ & 1.28 & 20.16 & 269.9 & 7.1 & 49 & B3135 \\
\hline 25 & 10:08:50.70 & $+12: 17: 46.9$ & 1.15 & 20.31 & 270.5 & 6.7 & 35 & K677 \\
\hline 26 & 10:07:55.77 & $+12: 17: 55.2$ & 1.14 & 20.48 & 277.3 & 6.0 & 47 & \\
\hline 27 & 10:08:01.84 & $+12: 17: 56.6$ & 1.43 & 19.82 & 275.4 & 5.4 & 61 & B2488 \\
\hline 28 & $10: 08: 45.25$ & $+12: 17: 57.8$ & 1.28 & 20.08 & 248.9 & 5.5 & 52 & \\
\hline 29 & $10: 08: 38.58$ & $+12: 18: 21.0$ & 0.94 & 19.71 & 274.5 & 8.3 & 41 & \\
\hline 30 & $10: 08: 56.58$ & $+12: 18: 22.4$ & 1.13 & 20.22 & 271.1 & 0.3 & 30 & \\
\hline 31 & 10:08:34.85 & $+12: 18: 22.6$ & 1.24 & 19.98 & 256.2 & 0.1 & 47 & \\
\hline 32 & 10:08:15.96 & $+12: 18: 25.3$ & 1.17 & 20.25 & 282.9 & 3.0 & 34 & \\
\hline 33 & $10: 07: 57.28$ & $+12: 18: 26.1$ & 1.40 & 19.74 & 284.3 & 7.5 & 69 & \\
\hline 34 & 10:08:44.09 & $+12: 18: 29.0$ & 1.13 & 19.66 & 278.8 & 9.5 & 42 & \\
\hline 35 & 10:08:47.87 & $+12: 18: 29.9$ & 1.46 & 19.70 & 248.8 & 8.0 & 39 & \\
\hline 36 & $10: 08: 36.01$ & $+12: 18: 32.6$ & 1.30 & 19.58 & 254.0 & 0.6 & 45 & \\
\hline 37 & 10:07:59.36 & $+12: 18: 35.7$ & 1.60 & 19.75 & 273.9 & 6.3 & 54 & \\
\hline 38 & 10:08:58.70 & $+12: 18: 37.1$ & 1.44 & 19.85 & 257.2 & 8.2 & 46 & \\
\hline 39 & 10:08:41.92 & $+12: 18: 39.7$ & 1.16 & 20.22 & 250.4 & 7.8 & 31 & B25820 \\
\hline 40 & $10: 08: 51.73$ & $+12: 18: 45.7$ & 1.28 & 20.23 & 278.9 & 3.0 & 49 & \\
\hline 41 & $10: 08: 20.21$ & $+12: 18: 46.6$ & 1.41 & 19.62 & 258.0 & 0.1 & 27 & \\
\hline 42 & $10: 08: 33.75$ & $+12: 18: 47.0$ & 1.26 & 19.23 & 275.4 & 0.0 & 50 & \\
\hline 43 & $10: 08: 24.22$ & $+12: 18: 53.3$ & 1.38 & 19.93 & 259.0 & 2.4 & 42 & \\
\hline 44 & 10:08:40.60 & $+12: 19: 02.8$ & 1.25 & 19.66 & 276.9 & 2.7 & 48 & \\
\hline 45 & 10:08:30.67 & $+12: 19: 30.0$ & 1.00 & 20.34 & 259.4 & 4.6 & 39 & \\
\hline 46 & $10: 08: 21.15$ & $+12: 19: 43.6$ & 1.52 & 19.72 & 270.8 & 1.0 & 35 & \\
\hline 47 & 10:08:39.42 & $+12: 20: 05.9$ & 1.16 & 19.74 & 271.2 & 3.8 & 51 & \\
\hline 48 & $10: 08: 37.29$ & $+12: 20: 12.2$ & 1.24 & 19.88 & 270.7 & 1.3 & 43 & K351 \\
\hline 49 & 10:08:13.35 & $+12: 20: 13.8$ & 1.27 & 19.64 & 292.2 & 2.9 & 55 & \\
\hline 50 & 10:08:22.17 & $+12: 20: 14.9$ & 1.38 & 19.15 & 289.8 & 0.9 & 59 & \\
\hline 51 & 10:08:28.41 & $+12: 20: 29.6$ & 1.51 & 19.61 & 267.6 & 2.1 & 64 & K137 \\
\hline 52 & 10:08:14.96 & $+12: 20: 43.9$ & 1.47 & 19.58 & 266.6 & 2.9 & 50 & \\
\hline 53 & 10:08:19.17 & $+12: 20: 48.9$ & 1.38 & 19.87 & 255.3 & 2.0 & 38 & B8203 \\
\hline 54 & 10:08:18.24 & $+12: 20: 54.6$ & 1.27 & 20.02 & 268.0 & 0.7 & 31 & \\
\hline 101 & $10: 07: 51.18$ & $+12: 17: 36.6$ & 1.52 & 19.56 & 49.6 & 8.5 & 72 & \\
\hline 102 & 10:08:05.23 & $+12: 18: 13.5$ & 1.05 & 19.96 & -27.9 & 5.5 & 52 & \\
\hline 103 & $10: 08: 53.43$ & $+12: 18: 27.4$ & 1.36 & 19.88 & 90.4 & 8.7 & 55 & \\
\hline
\end{tabular}

Notes. $v$ is the heliocentric radial velocity and $\Delta v$ the absolute semi-difference in radial velocity of the individual spectra. The last column gives the identification of stars in common with Bosler et al. (2007) and Koch et al. (2007b).

the two values independently measured from the single spectra. The results, corrected to heliocentric velocities using the rvcorr task, are given in Table 1. The distribution of radial velocities, shown in Fig. 4, is well fitted by a Gaussian function centred at
$271 \mathrm{~km} \mathrm{~s}^{-1}$ with a dispersion $13.7 \mathrm{~km} \mathrm{~s}^{-1}$. All but three stars have heliocentric radial velocities within $3 \sigma$ of the peak, and therefore are considered members of Leo I (members have ID $<100$ in Table 1). 


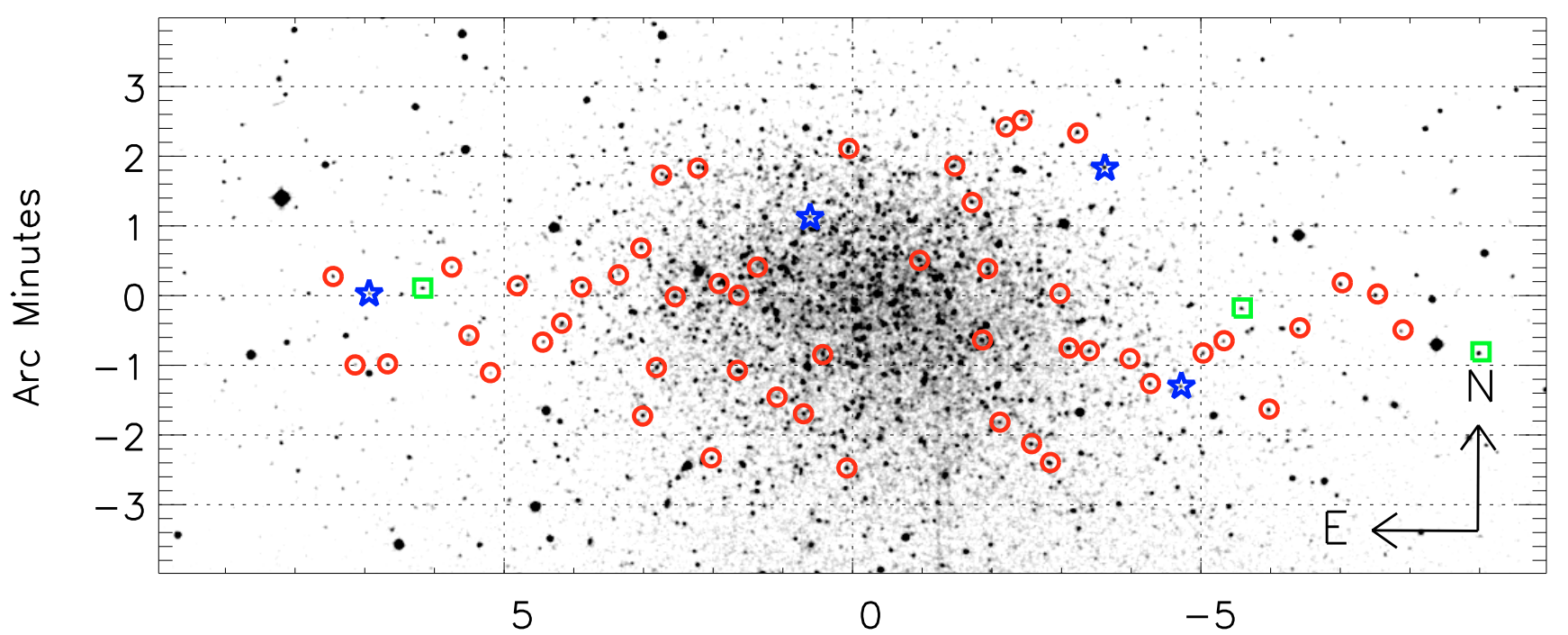

Arc Minutes

Fig. 1. Digitized Sky Survey image of the Leo I field, centred at $10^{\mathrm{h}} 08^{\mathrm{m}} 28^{\mathrm{s}} .1,+12^{\circ} 18^{\prime} 23^{\prime \prime}(\mathrm{J} 2000)$. Starred symbols mark the 4 metal-poor member stars, the circles are other member stars, squares represent interlopers.

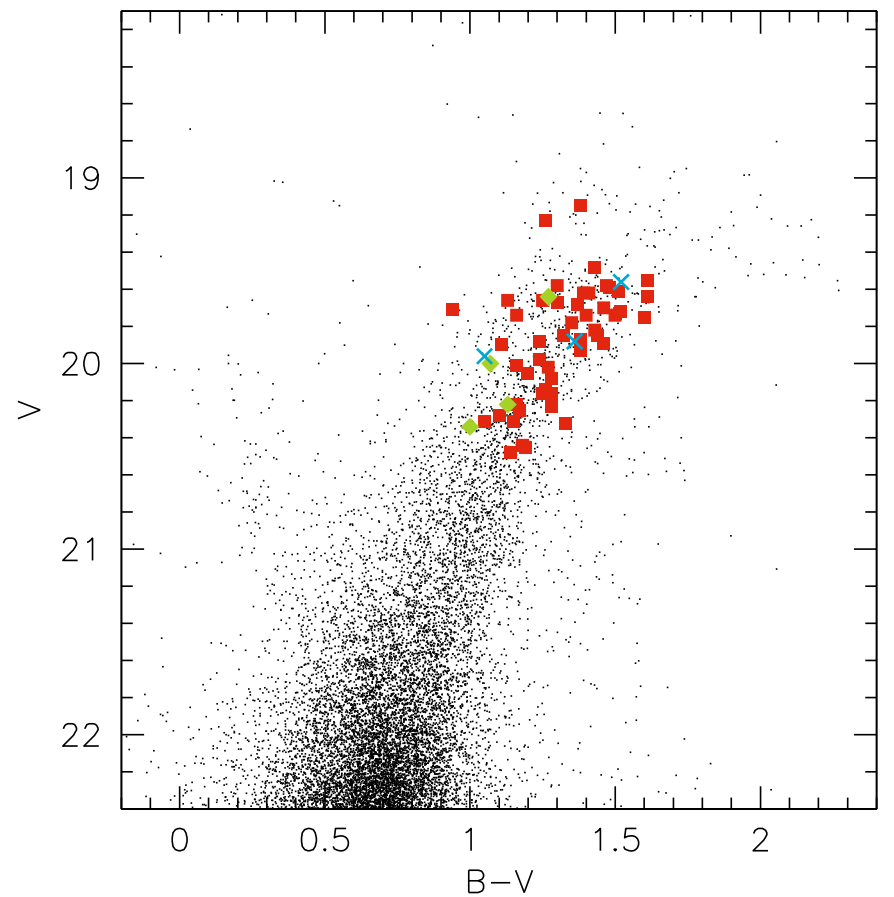

Fig. 2. Target stars in the colour-magnitude diagram of Leo I. The filled squares represent red giant stars in Leo I, the diamonds are the 4 metalpoor red giant members, while crosses mark non-members.

Radial velocity errors (given in Col. 7 of Table 1) were estimated by calculating the differences between radial velocities measured on the two individual spectra. The distribution of the differences is approximately Gaussian with a mean $-6.4 \mathrm{~km} \mathrm{~s}^{-1}$ and dispersion $10.4 \mathrm{~km} \mathrm{~s}^{-1}$. The mean velocity difference provides a good estimate of the mask-to-mask systematic errors, which include wavelength calibration errors and the centring errors of the targets on the slitlets, while the standard deviation represents a combination of random errors. We assume $\sigma_{v}=$ $\sigma_{v_{1}-v_{2}} / \sqrt{2}=7.4 \mathrm{~km} \mathrm{~s}^{-1}$ as a good approximation to the standard error of the radial velocity measured on the combined spectrum.
Table 2. Log of the observations.

\begin{tabular}{lrl}
\hline \hline Field & \multicolumn{1}{c}{ Night } & $t_{\exp }(\mathrm{s})$ \\
\hline NGC 4590 & 6 May 2002 & $15+60$ \\
NGC 5927 & 6 May 2002 & $60+300$ \\
NGC 6171 & 6 May 2002 & $10+60$ \\
NCG 5904 & 3 May 2003 & $60+300$ \\
NGC 6397 & 5 May 2003 & $60+300$ \\
NGC 6528 & 5 May 2003 & $60+300$ \\
NGC 4372 & 24 May 2003 & $60+300$ \\
NGC 6752 & 24 May 2003 & $60+300$ \\
Leo I field U & 4 May 2003 & $2 \times 2870$ \\
Leo I field R & 20, 21 Dec. 2003 & $2 \times 2870$ \\
Leo I field D & 22 May 2003 & $2 \times 2870$ \\
Leo I field L & 16, 25 Jun. 2003 & $2 \times 2870$ \\
\hline
\end{tabular}

Therefore our systemic radial velocity of Leo I is $271 \pm 6.4$ (systematic) \pm 7.4 (random) $\mathrm{km} \mathrm{s}^{-1}$. Our error estimates are consistent with the $0.85 \AA$ pixel $^{-1}$ spectral resolution provided by our instrumental setup, which corresponds to a resolution in radial velocity of $\sim 30 \mathrm{~km} \mathrm{~s}^{-1}$ pixel $^{-1}$ in the CaT wavelength range. Koch et al. (2007b) measured $284.2 \mathrm{~km} \mathrm{~s}^{-1}$ with a velocity dispersion of $9.9 \mathrm{~km} \mathrm{~s}^{-1}$, while $282.6 \pm 9.8 \mathrm{~km} \mathrm{~s}^{-1}$ was the value measured by Bosler et al. (2007). Most recently, Mateo et al. (2008) obtained a mean heliocentric velocity $282.9 \pm 0.5 \mathrm{~km} \mathrm{~s}^{-1}$ and a dispersion $9.2 \pm 0.4 \mathrm{~km} \mathrm{~s}^{-1}$ from echelle spectroscopy of 328 Leo I members. The systematic difference of $\sim 10 \mathrm{~km} \mathrm{~s}^{-1}$ between our mean velocity estimate and previous results is consistent with pointing errors. In the CaT wavelength range, a velocity of $10 \mathrm{~km} \mathrm{~s}^{-1}$ corresponds to a shift of $\sim 1 / 3$ pixel on the detector (see above), or 0.'08 on the sky.

\section{Equivalent widths and metallicity}

\subsection{Equivalent width measurements}

We measured the equivalent widths $(E W \mathrm{~s})$ of CaT lines in the spectra of target stars in Leo I and the calibrating GCs as follows. We first normalised the spectra over a wavelength interval encompassing, for each line, the side bands defined by 


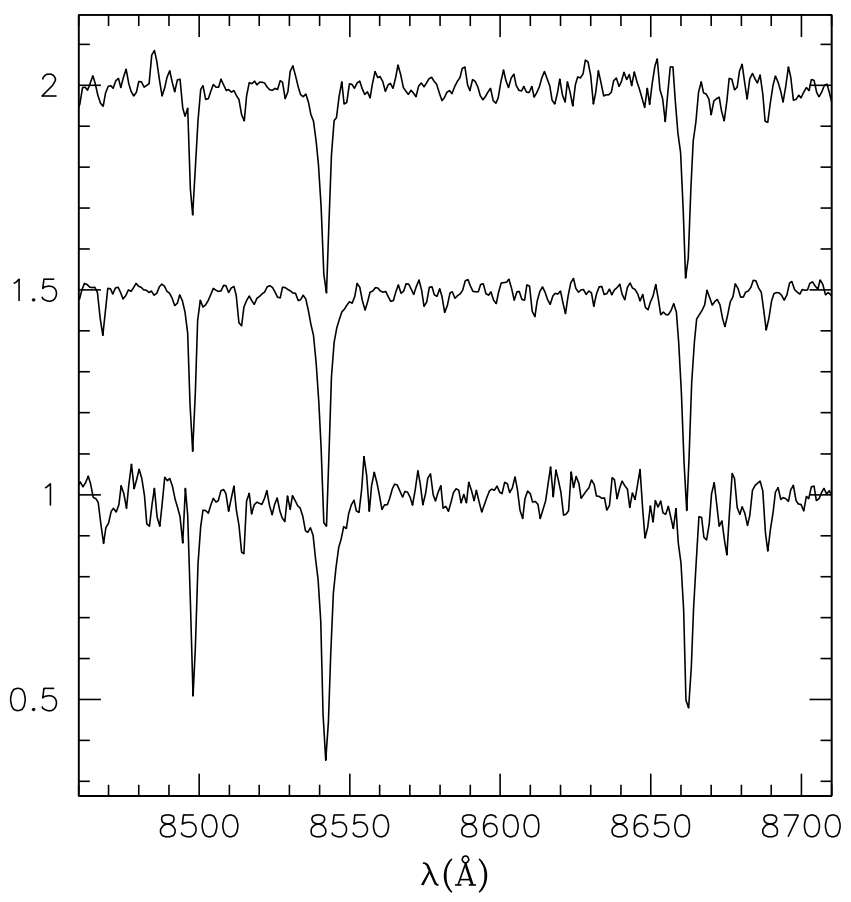

Fig. 3. Examples of normalised, background-subtracted spectra of RGB stars in Leo I (stars \#17, \#19, \#54, from top to bottom). The spectra are shown on a rest-frame wavelength scale and vertically shifted for clarity.

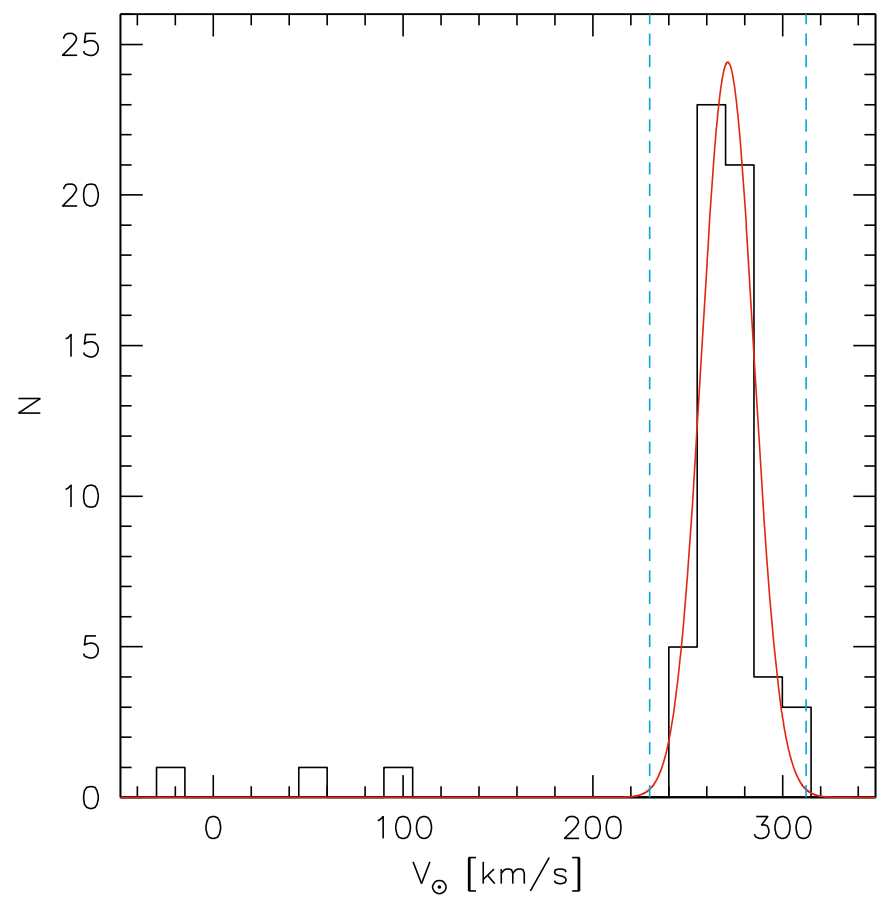

Fig. 4. Heliocentric radial velocities of stars in the Leo I field. The distribution is fitted with a Gaussian centred at $271 \mathrm{~km} \mathrm{~s}^{-1}$ and with dispersion $13.7 \mathrm{~km} \mathrm{~s}^{-1}$. The vertical dashed lines represents the $3 \sigma$ limits used to select members of Leo I.

Armandroff \& Da Costa (1991). Equivalent widths were then measured for the two stronger CaT lines in the co-added spectra by fitting a model profile over the line central bandpasses as defined by the same authors. Following Cole et al. (2004), the fitted model is the sum of a Gaussian and a Lorentzian profiles with a common line centre $\lambda_{m}$,

$F(\lambda)=1-A_{G} \exp \left[-\frac{\left(\lambda-\lambda_{m}\right)^{2}}{2 \sigma^{2}}\right]-A_{L}\left[\frac{\left(\lambda-\lambda_{m}\right)^{2}}{\Gamma^{2}}+1\right]^{-1}$

with the best-fit parameters determined using a LevenbergMarquardt least-squares algorithm (coded in an IDL procedure by Markwardt ${ }^{2}$. The Ca II line strength was then defined as the unweighted sum of the two equivalent widths,

$\Sigma W=E W_{8542}+E W_{8662}$

The sum equivalent widths are given in Table 3 for the red giants in Leo I and in Table 4 for the RGB stars in the template GCs.

To estimate the equivalent width measurement errors, we also measured the CaT line strengths independently on the individual spectra of each star in Leo I. A comparison of the two $\Sigma W$ measurements is shown in Fig. 5, where they appear to be well correlated. The histogram of the differences $\Delta_{\Sigma W}=$ $\left(\Sigma W_{2}-\Sigma W_{1}\right)$ (shown in Fig. 5, lower panel) is well fitted by a Gaussian function centred at $\left\langle\Delta_{\Sigma W}\right\rangle=0.03$ and with a standard deviation $\sigma_{\Delta \Sigma W}=0.44 \AA$. Since the two individual spectra have comparable $S / N$ ratio, we adopt $\sigma_{\Sigma W}=\sigma_{\Delta \Sigma W} / \sqrt{2}=0.31 \AA$ as our error estimate for $\Sigma W$ measured on the combined spectrum. For comparison, the half-range $\epsilon_{\Sigma W}=\left|\Delta_{\Sigma W} / 2\right|$ is listed for each Leo I star in Table 3.

\subsection{Reduced EW}

According to the CaT method (Armandroff \& Da Costa 1991), the gravity and $T_{\text {eff }}$ dependence of CaT lines is accounted for by introducing a linear correction to the line strengths which depends on the star's luminosity, that is

$W^{\prime}=\Sigma W+\beta\left(V-V_{\mathrm{HB}}\right)$

where $\beta$ is a constant and $\left(V-V_{\mathrm{HB}}\right)$ is the difference between the magnitude of the star and the horizontal branch (HB) in the $V$ band. In globular clusters, this reduced equivalent width $W^{\prime}$ was found to be well correlated with metallicity (Armandroff \& Zinn 1988; Armandroff \& Da Costa 1991; Rutledge et al. 1997b). This provides the empirical basis for the validity of the CaT method. Applied to composite stellar populations, the method is less straightforward and has been widely discussed in the recent literature. We will return to this point later on.

In Fig. 6, we plot the sum of equivalent widths $\Sigma W$ versus $V-V_{\mathrm{HB}}$ for all stars with good $S / N$ spectra, both in Leo I and the template GCs. The magnitude of the HB (of old stars) in Leo I, $V_{\mathrm{HB}}=22.60$, is from Held et al. (2001), while for the template GCs, $V_{\mathrm{HB}}$ was taken from Rutledge et al. (1997a) (listed in Table 5 together with clusters' metallicities). For all the globular clusters, our CaT line strengths define clean, well separated linear sequences generally consistent with a constant slope and having different, metallicity-dependent zero points. In this diagram, the Leo I stars show quite a large dispersion, although most of them are located between the sequences of NGC 6397 and M5. This spread in CaT line strengths is real, being larger than the typical measurement error $\sigma_{\Sigma W} \approx 0.3 \AA$. By assuming a common slope and lumping together the data for all globular clusters, we derived a slope $\beta=0.627 \pm 0.021$. This value can only be compared with previous results that use the same definition of $\Sigma W$. This is the case for Tolstoy et al. (2001), who found $\beta=0.64 \pm 0.02$, in agreement with our result.

\footnotetext{
${ }^{2}$ http://cow.physics.wisc.edu/ craigm/idl/idl.html
} 
Table 3. Measurements of metallicity and age for stars in Leo I.

\begin{tabular}{|c|c|c|c|c|c|c|c|c|c|}
\hline ID & $\begin{array}{l}\Sigma W \\
(\AA)\end{array}$ & $\begin{array}{l}\epsilon_{\Sigma W} \\
(\AA)\end{array}$ & $\begin{array}{c}W^{\prime} \\
(\AA)\end{array}$ & {$[\mathrm{Fe} / \mathrm{H}]^{\mathrm{ZW}}$} & {$[\mathrm{Fe} / \mathrm{H}]^{\mathrm{CG}}$} & {$[\mathrm{M} / \mathrm{H}]$} & $\begin{array}{r}\text { Age } \\
\text { (Gyr) }\end{array}$ & $\begin{array}{c}\Delta \mathrm{age}^{-} \\
(\mathrm{Gyr})\end{array}$ & $\begin{array}{c}\Delta \mathrm{age}^{+} \\
(\mathrm{Gyr})\end{array}$ \\
\hline 1 & 5.58 & 0.11 & 3.71 & -1.55 & -1.41 & -1.21 & 3.4 & 0.8 & 1.1 \\
\hline 2 & 6.12 & 0.38 & 4.23 & -1.28 & -1.16 & -0.98 & 2.8 & 0.6 & 1.1 \\
\hline 3 & 4.85 & 0.29 & 3.16 & -1.78 & -1.64 & -1.44 & 10.5 & 1.8 & 1.8 \\
\hline 4 & 5.77 & 0.04 & 3.86 & -1.48 & -1.34 & -1.15 & 5.5 & 1.2 & 0.9 \\
\hline 5 & 5.30 & 0.19 & 3.85 & -1.48 & -1.35 & -1.15 & 2.1 & 0.5 & 0.8 \\
\hline 6 & 5.92 & 0.21 & 4.16 & -1.32 & -1.19 & -1.01 & 2.6 & 0.7 & 0.8 \\
\hline 7 & 4.29 & 0.22 & 2.56 & -1.97 & -1.84 & -1.65 & 8.8 & 1.7 & 2.7 \\
\hline 8 & 4.98 & 0.19 & 3.19 & -1.77 & -1.63 & -1.43 & 9.8 & 3.0 & 0.9 \\
\hline 9 & 5.20 & 0.19 & 3.85 & -1.48 & -1.35 & -1.15 & 5.4 & 2.0 & 1.3 \\
\hline 10 & 2.45 & 0.06 & 0.82 & -2.17 & -2.14 & -2.04 & 6.3 & 1.6 & 1.9 \\
\hline 11 & 4.45 & 0.17 & 2.85 & -1.89 & -1.75 & -1.55 & 6.4 & 1.7 & 2.0 \\
\hline 12 & 4.92 & 0.04 & 3.57 & -1.62 & -1.48 & -1.28 & 6.1 & 2.1 & 2.7 \\
\hline 13 & 5.43 & 0.08 & 3.81 & -1.50 & -1.37 & -1.17 & 2.1 & 0.5 & 0.8 \\
\hline 14 & 5.81 & 0.38 & 3.98 & -1.42 & -1.28 & -1.10 & 2.7 & 0.6 & 0.8 \\
\hline 15 & 4.20 & 0.08 & 2.84 & -1.89 & -1.76 & -1.55 & 9.4 & 2.2 & 2.4 \\
\hline 16 & 5.30 & 0.05 & 3.76 & -1.53 & -1.39 & -1.19 & 5.2 & 1.1 & 2.1 \\
\hline 17 & 4.04 & 0.03 & 2.60 & -1.96 & -1.83 & -1.63 & 4.2 & 1.1 & 1.6 \\
\hline 18 & 6.00 & 0.23 & 4.16 & -1.32 & -1.19 & -1.01 & 2.0 & 0.4 & 0.3 \\
\hline 19 & 5.25 & 0.02 & 3.72 & -1.55 & -1.41 & -1.21 & 5.6 & 1.5 & 2.6 \\
\hline 20 & 4.95 & 0.20 & 3.26 & -1.74 & -1.60 & -1.40 & 1.9 & 0.3 & 0.4 \\
\hline 21 & 5.47 & 0.51 & 3.51 & -1.64 & -1.50 & -1.30 & 3.4 & 0.7 & 0.9 \\
\hline 22 & 5.02 & 0.14 & 3.59 & -1.61 & -1.47 & -1.27 & 11.2 & 1.7 & 1.4 \\
\hline 23 & 6.20 & 0.03 & 4.34 & -1.22 & -1.10 & -0.92 & 4.3 & 1.1 & 1.9 \\
\hline 24 & 5.70 & 0.13 & 4.17 & -1.32 & -1.19 & -1.00 & 3.3 & 1.0 & 1.5 \\
\hline 25 & 5.11 & 0.39 & 3.67 & -1.57 & -1.43 & -1.23 & 4.1 & 1.9 & 2.0 \\
\hline 26 & 4.83 & 0.11 & 3.50 & -1.64 & -1.51 & -1.30 & 5.8 & 2.0 & 2.1 \\
\hline 27 & 5.57 & 0.02 & 3.83 & -1.49 & -1.36 & -1.16 & 5.7 & 1.7 & 1.8 \\
\hline 28 & 5.40 & 0.29 & 3.82 & -1.50 & -1.36 & -1.17 & 4.6 & 1.3 & 1.5 \\
\hline 29 & 4.93 & 0.05 & 3.12 & -1.80 & -1.66 & -1.45 & .. & $\ldots$ & $\ldots$ \\
\hline 30 & 2.24 & 0.00 & 0.75 & -2.17 & -2.14 & -2.05 & 10.2 & 2.0 & 1.0 \\
\hline 31 & 5.36 & 0.46 & 3.72 & -1.55 & -1.41 & -1.21 & 3.2 & 0.7 & 1.4 \\
\hline 32 & 4.53 & 0.41 & 3.05 & -1.82 & -1.68 & -1.48 & 6.3 & 2.0 & 3.0 \\
\hline 33 & 4.90 & 0.01 & 3.10 & -1.80 & -1.66 & -1.46 & 8.5 & 2.0 & 2.2 \\
\hline 34 & 5.13 & 0.29 & 3.29 & -1.73 & -1.59 & -1.39 & 1.7 & 0.2 & 0.3 \\
\hline 35 & 5.73 & 0.03 & 3.91 & -1.45 & -1.32 & -1.13 & 4.9 & 1.2 & 1.7 \\
\hline 36 & 5.46 & 0.07 & 3.57 & -1.61 & -1.48 & -1.27 & 2.6 & 0.5 & 0.8 \\
\hline 37 & 5.50 & 0.01 & 3.71 & -1.55 & -1.41 & -1.22 & 10.2 & 2.5 & 1.9 \\
\hline 38 & 5.40 & 0.33 & 3.68 & -1.57 & -1.43 & -1.23 & 7.1 & 1.9 & 1.8 \\
\hline 39 & 5.32 & 0.48 & 3.82 & -1.50 & -1.36 & -1.17 & 2.5 & 0.7 & 1.3 \\
\hline 40 & 5.15 & 0.11 & 3.66 & -1.57 & -1.44 & -1.24 & 7.2 & 2.0 & 2.7 \\
\hline 41 & 5.08 & 0.05 & 3.21 & -1.76 & -1.62 & -1.42 & 5.5 & 1.3 & 2.0 \\
\hline 42 & 6.45 & 0.03 & 4.34 & -1.22 & -1.10 & -0.92 & & $\cdots$ & . \\
\hline 43 & 6.12 & 0.12 & 4.45 & -1.16 & -1.04 & -0.87 & 2.8 & 0.8 & 1.2 \\
\hline 44 & 5.28 & 0.21 & 3.44 & -1.67 & -1.53 & -1.33 & 2.6 & 0.7 & 0.7 \\
\hline 45 & 2.94 & 0.23 & 1.52 & -2.16 & -2.07 & -1.92 & 4.9 & 1.5 & 1.9 \\
\hline 46 & 6.13 & 0.07 & 4.33 & -1.23 & -1.10 & -0.93 & 3.8 & 1.1 & 1.3 \\
\hline 47 & 5.86 & 0.44 & 4.07 & -1.37 & -1.24 & -1.05 & 1.5 & 0.1 & 0.2 \\
\hline 48 & 6.21 & 0.21 & 4.51 & -1.12 & -1.00 & -0.84 & 1.7 & 0.2 & 0.2 \\
\hline 49 & 3.26 & 0.26 & 1.41 & -2.16 & -2.09 & -1.95 & 8.0 & 1.9 & 1.9 \\
\hline 50 & 5.81 & 0.04 & 3.65 & -1.58 & -1.44 & -1.24 & & $\ldots$ & $\ldots$ \\
\hline 51 & 4.96 & 0.06 & 3.09 & -1.81 & -1.67 & -1.47 & 7.8 & 1.3 & 1.0 \\
\hline 52 & 5.79 & 0.38 & 3.89 & -1.46 & -1.33 & -1.13 & 4.0 & 1.0 & 1.1 \\
\hline 53 & 5.67 & 0.11 & 3.96 & -1.43 & -1.29 & -1.10 & 4.6 & 1.4 & 2.4 \\
\hline 54 & 6.17 & 0.22 & 4.55 & -1.09 & -0.98 & -0.81 & 1.9 & 0.3 & 0.7 \\
\hline
\end{tabular}

Notes. $\epsilon_{\Sigma W}$ is the absolute semi-difference of the equivalent widths measured on the individual spectra. The listed metallicity values were calculated from $W^{\prime}$ using the quadratic calibration. The last two columns give the lower and upper confidence intervals of our age estimates (see text for details).

\subsection{Metallicity calibration}

Using our reduced CaT equivalent widths $W^{\prime}$ and the published metallicities for the Galactic globular clusters, we re-determined the calibration relations between $W^{\prime}$ and metallicity on
3 different abundance scales: the $[\mathrm{Fe} / \mathrm{H}]$ scales of Zinn \& West (1984) and Carretta \& Gratton (1997) and the global metallicity $[\mathrm{M} / \mathrm{H}]$, as defined by Salaris et al. (1993). The metallicities of the GCs (Table 5) were taken from Ferraro et al. (1999), except for the metal-rich cluster NGC 6528, for which 
Table 4. Observed stars in template globular clusters.

\begin{tabular}{|c|c|c|c|c|}
\hline Cluster & ID & V & $B-V$ & $\Sigma W(\AA)$ \\
\hline \multirow[t]{10}{*}{ M 5} & III-45 & 14.75 & 0.82 & 4.62 \\
\hline & II-50 & 13.92 & 0.96 & 5.12 \\
\hline & II-51 & 14.05 & 0.96 & 4.90 \\
\hline & II- 80 & 14.31 & 0.91 & 4.89 \\
\hline & II-74 & 13.82 & 1.01 & 5.05 \\
\hline & I-2 & 13.87 & 1.02 & 5.24 \\
\hline & I-50 & 13.91 & 0.97 & 5.06 \\
\hline & I-61 & 13.37 & 1.17 & 5.40 \\
\hline & I-68 & 12.37 & 1.52 & 6.25 \\
\hline & I-71 & 13.01 & 1.29 & 5.57 \\
\hline \multirow[t]{11}{*}{ NGC 4372} & 20 & 12.88 & 1.57 & 3.78 \\
\hline & 14 & 14.29 & 1.29 & 2.88 \\
\hline & 13 & 12.72 & 1.73 & 4.13 \\
\hline & 10 & 13.82 & 1.29 & 3.00 \\
\hline & 95 & 14.48 & 1.30 & 2.72 \\
\hline & 91 & 14.45 & 1.33 & 2.81 \\
\hline & 89 & 14.49 & 1.29 & 2.83 \\
\hline & 141 & 12.93 & 1.65 & 4.01 \\
\hline & 74 & 14.17 & 1.40 & 3.09 \\
\hline & 76 & 14.18 & 1.30 & 3.16 \\
\hline & 77 & 14.19 & 1.28 & 3.07 \\
\hline \multirow[t]{4}{*}{ NGC 6171} & 62 & 13.97 & 1.62 & 5.83 \\
\hline & 100 & 14.21 & 1.40 & 5.50 \\
\hline & I & 13.89 & 1.46 & 5.66 \\
\hline & $\mathrm{F}$ & 13.39 & 1.70 & 6.21 \\
\hline \multirow[t]{5}{*}{ NGC 6397} & 328 & 12.07 & 0.93 & 3.19 \\
\hline & 326 & 12.78 & 0.89 & 2.89 \\
\hline & 337 & 12.58 & 0.90 & 2.83 \\
\hline & 343 & 11.42 & 1.13 & 3.34 \\
\hline & 361 & 11.67 & 1.08 & 3.18 \\
\hline \multirow[t]{3}{*}{ NGC 6528} & R2-8 & 15.79 & 1.89 & 6.80 \\
\hline & R1-42 & 16.46 & 1.62 & 6.34 \\
\hline & R2-41 & 16.30 & 1.64 & 6.44 \\
\hline \multirow[t]{5}{*}{ NGC 6752} & 4 & 13.70 & 0.85 & 3.86 \\
\hline & 8 & 11.96 & 1.05 & 4.99 \\
\hline & 28 & 13.17 & 0.89 & 4.18 \\
\hline & 29 & 11.79 & 1.17 & 4.98 \\
\hline & 30 & 12.15 & 1.12 & 4.83 \\
\hline \multirow[t]{5}{*}{ NGC 5927} & 133 & 14.75 & 1.97 & 6.45 \\
\hline & 372 & 14.66 & 2.11 & 6.28 \\
\hline & 335 & 14.44 & 1.94 & 6.63 \\
\hline & 190 & 14.29 & 2.02 & 6.71 \\
\hline & 65 & 14.64 & 1.92 & 6.58 \\
\hline \multirow[t]{6}{*}{ NGC 4590} & 144 & 12.80 & 1.29 & 3.72 \\
\hline & 239 & 14.19 & 0.87 & 2.88 \\
\hline & II72 & 15.03 & 0.85 & 2.60 \\
\hline & 30 & 14.15 & 0.87 & 2.64 \\
\hline & 74 & 14.59 & 0.84 & 2.36 \\
\hline & 119 & 13.62 & 0.95 & 2.90 \\
\hline
\end{tabular}

Notes. The IDs, magnitudes, and colours of stars are those given in the original photometry papers quoted by Rutledge et al. (1997a).

the more recent results of Zoccali et al. (2004) were adopted $([\mathrm{Fe} / \mathrm{H}]=-0.1,[\alpha / \mathrm{Fe}]=0.1)$. The global metallicity of NGC $6528([\mathrm{M} / \mathrm{H}]=-0.03)$ was calculated using the relation from Salaris et al. (1993):

$[\mathrm{M} / \mathrm{H}]=[\mathrm{Fe} / \mathrm{H}]+\log \left(0.683 f_{\alpha}+0.362\right)$

$\log f_{\alpha}=[\alpha / \mathrm{Fe}]$.

Figure 7 shows the $W^{\prime}$-metallicity relations for the three scales, along with quadratic fits to the whole dataset and linear fits to the metal-poor and intermediate globular clusters. The quadratic relations provide a better fit to the GC metallicities over the whole metallicity range of template GCs. The curvature is driven
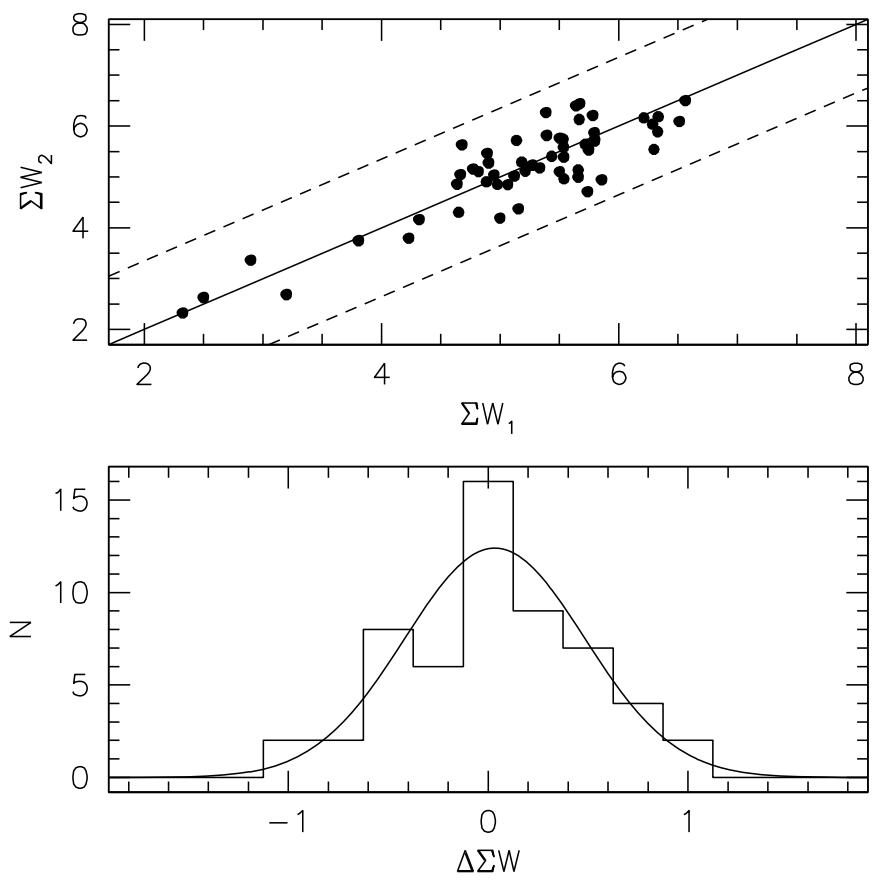

Fig. 5. Upper panel: correlation between two independent $E W$ measurements on individual spectra of Leo I stars. The dashed lines show the $3 \sigma$ interval. Lower panel: histogram of the differences between the two measurements, fitted by a Gaussian profile with $\sigma_{\Delta \Sigma W}=0.44 \AA$.

by the data for two most metal-rich globular clusters, consistently with a fall in sensitivity of the Ca II index at high metallicity. Previous studies which included metal-rich GCs also found quadratic relations (Armandroff \& Da Costa 1991; Da Costa \& Armandroff 1995; Carretta et al. 2001; Bosler et al. 2007). Linear relations have been proposed by other studies (most recently, Cole et al. 2004; Koch et al. 2007b; Carrera et al. 2007) using metal-rich open clusters to constrain the metal-rich end of the $W^{\prime}-[\mathrm{Fe} / \mathrm{H}]$ relation. A full discussion of the behaviour of CaT line strengths against metallicity is beyond the scope of this paper, and will be presented in a future paper along with a large dataset of calibrating globular clusters. For our data, a linear relation indeed provides a good fit for stars less metal-rich than the template cluster NGC $6171([\mathrm{M} / \mathrm{H}]=-0.70)$ (Fig. 7). In the case of a metal-poor system such as Leo I, the linear and quadratic relations give similar results except for the most metal-poor stars. Our calibration is presently quite uncertain near the metal-poor end, being based on one globular cluster (NGC 4590). For this cluster, Pritzl et al. (2005) give a lower metallicity $([\mathrm{Fe} / \mathrm{H}] \sim$ -2.3) than that adopted in Table 5, yielding a better agreement with our linear calibration.

The main source of error on $[\mathrm{Fe} / \mathrm{H}]$ (or $[\mathrm{M} / \mathrm{H}]$ ) is the uncertainty on the measured equivalent width $\Sigma W$, since other sources of error, such as photometric errors for stars on the upper RGB of Leo I, the error on the HB level, or even the uncertainties associated to the fit parameters of the calibration relations, are negligible compared to the $\Sigma W$ measurement errors. A metallicity uncertainty can be computed for each star by error propagation using the values of $\epsilon_{\Sigma W}$ in Table 3 and the calibrations in Table 6. However, a more meaningful metallicity uncertainty is obtained using $\sigma_{W^{\prime}}$ as our estimate of the measurement error. For the quadratic $[\mathrm{M} / \mathrm{H}]$ calibration in Table $6, \sigma_{W^{\prime}}=0.31 \AA$ implies $\mathrm{a}[\mathrm{M} / \mathrm{H}]$ error $\sigma_{\mathrm{err}} \simeq 0.14$ dex for stars with $[\mathrm{M} / \mathrm{H}]=-1.20$.

The sources of uncertainty related to the CaT method itself are more difficult to quantify and predict. All traditional 


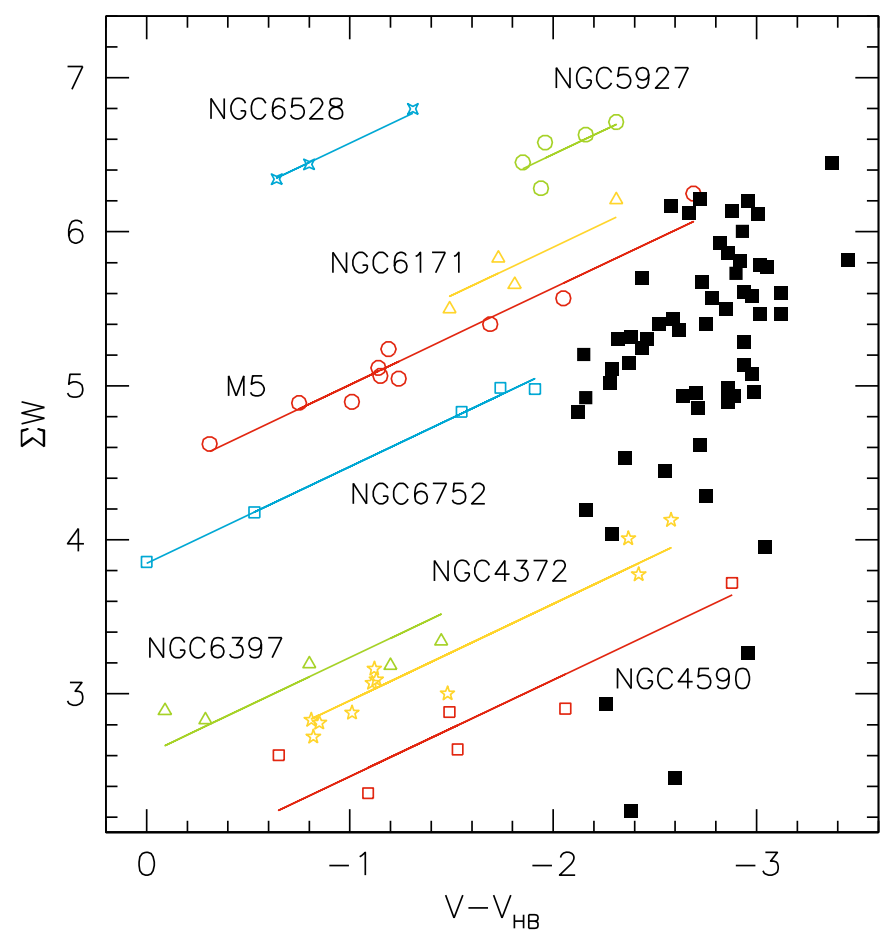

Fig. 6. The sum of the equivalent widths of the two most reliable $\mathrm{CaT}$ lines plotted as a function of the magnitude difference from the HB level. The calibration globular clusters are represented by open symbols (coded with different colours in the electronic edition of the journal). The metallicity of the clusters increases from the bottom to the top (see Table 5). The straight lines are best fits to the $E W$ s for each cluster, assuming a common best-fit slope. Filled squares refer to Leo I RGB stars.

Table 5. Parameters for the calibration Galactic globular clusters.

\begin{tabular}{lcccc}
\hline \hline Cluster & {$[\mathrm{Fe} / \mathrm{H}]_{\mathrm{ZW}}$} & {$[\mathrm{Fe} / \mathrm{H}]_{\mathrm{CG}}$} & {$[\mathrm{M} / \mathrm{H}]$} & $V_{\mathrm{HB}}$ \\
\hline NGC 6528 & -0.23 & -0.10 & -0.03 & 17.10 \\
NGC 5927 & -0.31 & -0.46 & -0.37 & 16.60 \\
NGC 6171 (M107) & -0.99 & -0.87 & -0.70 & 15.70 \\
NGC 5904 (M 5) & -1.40 & -1.11 & -0.90 & 15.06 \\
NGC 6752 & -1.54 & -1.42 & -1.21 & 13.70 \\
NGC 6397 & -1.91 & -1.82 & -1.65 & 12.87 \\
NGC 4372 & -2.08 & -1.94 & -1.74 & 15.30 \\
NGC 4590 (M68) & -2.09 & -1.99 & -1.81 & 15.68 \\
\hline
\end{tabular}

calibrations refer to Galactic globular clusters, which are simple and nearly coeval old stellar populations, and the applicability of these calibrations to complex stellar populations is not obvious. In our case, Leo I stars are on average several Gyr younger than those in GCs. At a given luminosity, a star in Leo I has a different mass from a GC star with the same metallicity and $V-V_{\mathrm{HB}}$. However, recent studies have shown that the CaT method can be used for complex stellar populations younger that those in globular clusters (Cole et al. 2004; Pont et al. 2004; Battaglia et al. 2008). Battaglia et al. (2008) have compared a linear metallicity calibration that uses the CaT of RGB stars in two dSph (Sculptor and Fornax) with spectroscopic $[\mathrm{Fe} / \mathrm{H}]$ values obtained from their high-resolution studies. The metallicities are in good agreement, although with some residual trends of about $0.1-0.2 \mathrm{dex}$, in the range $-2.5<[\mathrm{Fe} / \mathrm{H}]<-0.8$. These studies conclude that for ages older than $2.5 \mathrm{Gyr}$, the CaT line strengths are little affected by age, and suggest that the overall uncertainty related to age effects is $<0.2$ dex.

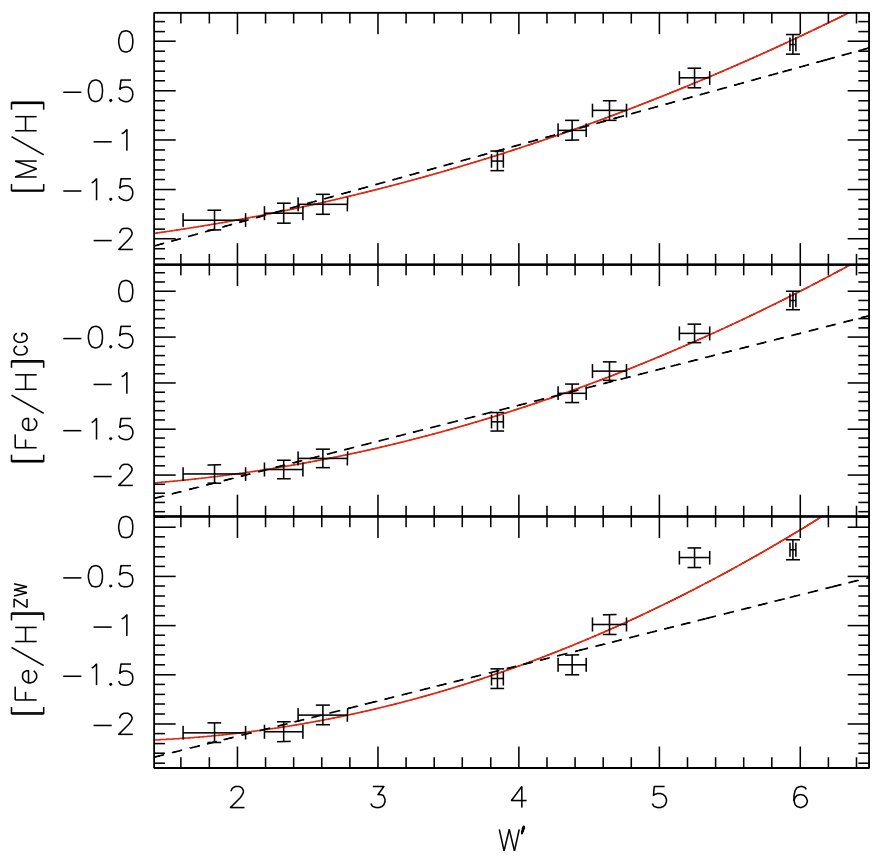

Fig. 7. The metallicity of the reference globular clusters against the reduced $E W$ of CaT lines, for three adopted metallicity scales. The solid lines are quadratic fits, while the dashed straight lines are linear fits obtained for clusters with $[\mathrm{Fe} / \mathrm{H}]<-0.6$.

Table 6. Metallicity calibrations.

\begin{tabular}{|c|c|c|}
\hline Type & & Calibration \\
\hline \multirow[t]{3}{*}{ quadratic } & {$[\mathrm{Fe} / \mathrm{H}]^{\mathrm{ZW}} \equiv$} & $\equiv 0.088 W^{\prime 2}-0.184 W^{\prime}-2.079$ \\
\hline & {$[\mathrm{Fe} / \mathrm{H}]^{\mathrm{CG}}=$} & $=0.072 W^{\prime 2}-0.076 W^{\prime}-2.122$ \\
\hline & {$[\mathrm{M} / \mathrm{H}]$} & $=0.051 W^{\prime 2}+0.056 W^{\prime}-2.125$ \\
\hline \multirow[t]{3}{*}{ linear } & {$[\mathrm{Fe} / \mathrm{H}]^{\mathrm{ZW}}=$} & $0.359 W^{\prime}-2.845$ \\
\hline & {$[\mathrm{Fe} / \mathrm{H}]^{\mathrm{CG}}=$} & $0.391 W^{\prime}-2.806$ \\
\hline & {$[\mathrm{M} / \mathrm{H}]$} & $0.395 W^{\prime}-2.628$ \\
\hline
\end{tabular}

\subsection{A new metallicity scale?}

Most CaT metallicity measurements in nearby galaxies use a $[\mathrm{Fe} / \mathrm{H}]$ scale based on observations of Galactic globular clusters (e.g. Pont et al. 2004; Cole et al. 2004; Battaglia et al. 2006; Koch et al. 2006). However, the relative abundances of $\alpha$ elements (including $\mathrm{Ca}$ ) in Local Group dwarfs are on average lower than in the Milky Way halo stars and GCs (Shetrone et al. 2001, 2003; Tolstoy et al. 2003; Geisler et al. 2005; Pritzl et al. 2005). To overcome this problem, Bosler et al. (2007) proposed a new calibration of $\mathrm{CaT}$ lines against the $[\mathrm{Ca} / \mathrm{H}]$ abundance, based on high-resolution spectroscopy of Galactic star clusters. In their hypothesis, the $[\mathrm{Ca} / \mathrm{H}]$ calibration is less affected by the difference in $[\mathrm{Ca} / \mathrm{Fe}]$ abundance ratios between red giant stars in globular clusters and dwarf spheroidal galaxies. However, the strength of CaT lines is also determined by other parameters (gravity and $T_{\text {eff }}$ ) in addition to $\mathrm{Ca}$ abundance. A comparison of $\mathrm{Ca}$ abundances derived from $\mathrm{CaT}$ lines with the results of highresolution abundance measurements for stars in two dSph galaxies (Battaglia et al. 2008) shows that, while the CaT lines trace both $\mathrm{Ca}$ and $\mathrm{Fe}$, their dependence on $\mathrm{Fe}$ abundance is stronger. Similarly, $[\mathrm{Ca} / \mathrm{H}]$ ratios derived from CaT lines for stars in Leo II dSph and globular clusters (Shetrone et al. 2009) systematically differ from those obtained from mid-resolution synthetic spectra, with a residual trend that is a function of metallicity. Since the effective temperature of red giants in globular clusters is driven 

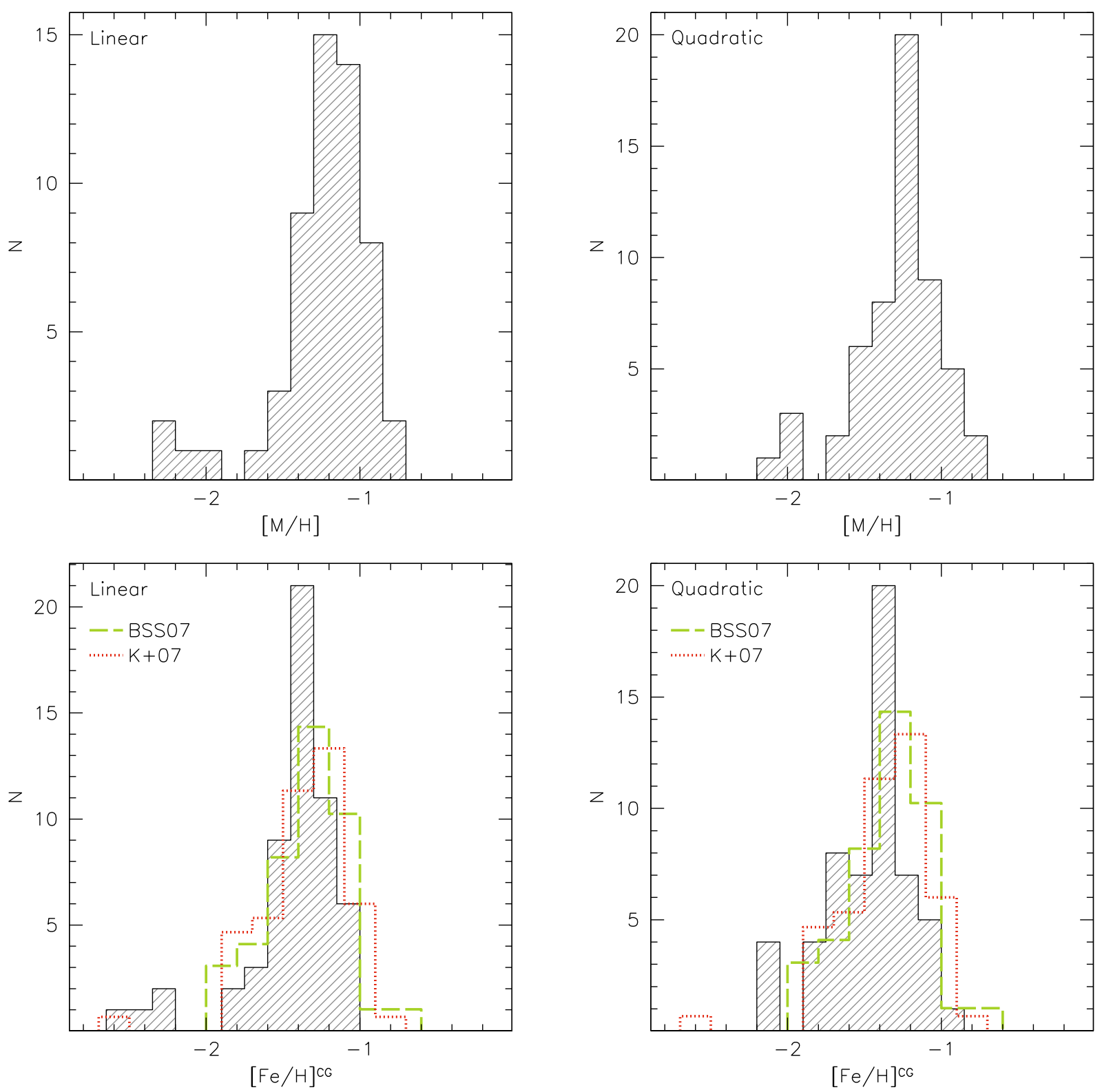

Fig. 8. Metallicity distribution of Leo I stars using a linear (left panels) or quadratic (right panels) calibration relation. In the upper panels we plot the MDF obtained with the $[\mathrm{M} / \mathrm{H}]$ calibration. The metallicity distributions on the $[\mathrm{Fe} / \mathrm{H}]$ scale of Carretta \& Gratton (1997) are shown in the lower panels, together with previous results from Bosler et al. (2007) and Koch et al. (2007b).

by their global metallicity [M/H] (Salaris et al. 1993), an empirical metallicity ranking based on $\mathrm{CaT}$ and a global metallicity $[\mathrm{M} / \mathrm{H}]$ scale, proposed here for the first time, appears to be the most empirically sound. In fact, $[\mathrm{M} / \mathrm{H}]$ (or, equivalently, $Z$ ) takes into account the abundances of both the $\alpha$-elements and Fe. Our data are therefore calibrated using the $[\mathrm{M} / \mathrm{H}]$ calibration in addition to the common $[\mathrm{Fe} / \mathrm{H}]$ scales.

\subsection{A concluding remark}

We conclude this section with a consideration that ought to be kept in mind throughout all the following discussion. While we give the metallicity of the stars in three flavours $\left([\mathrm{Fe} / \mathrm{H}]^{\mathrm{ZW}}\right.$, $[\mathrm{Fe} / \mathrm{H}]^{\mathrm{CG}}$, and $\left.[\mathrm{M} / \mathrm{H}]\right)$, this does not imply that we are determining the three metallicity parameters at the same time. The only observable quantity is the reduced equivalent width $W^{\prime}$. The calibration of $W^{\prime}$ in terms of metallicity relies on the assumption that $W^{\prime}$ is correlated with metallicity, i.e. a star in Leo I has the same metallicity as a star in a GC with the same $W^{\prime}$. The key questions are: what are the real drivers that determine the strength of the CaT lines? Do two stars with the same iron-tohydrogen ratio but different $\alpha$-elements composition have the same $W^{\prime}$ ? Some of these effects have been discussed by Battaglia et al. (2008), and are further addressed by a large observational program by our group whose results will be presented in future papers.

\section{The metallicity of Leo I stars}

\subsection{The observed metallicity distribution}

In Fig. 8 we show the metallicity distribution of Leo I red giant stars as derived from our data using both the $[\mathrm{Fe} / \mathrm{H}]$ metallicity scale of Carretta \& Gratton (1997) and the $[\mathrm{M} / \mathrm{H}]$ scale. The parameters of the distribution (mean and standard deviation, 
Table 7. Mean metallicity and standard deviation of red giants in Leo I.

\begin{tabular}{lrcc}
\hline \hline Scale & Fit & Mean & $\sigma$ \\
\hline$[\mathrm{Fe} / \mathrm{H}]^{\mathrm{ZW}}$ & linear & -1.53 & 0.17 \\
& quadratic & -1.55 & 0.21 \\
{$[\mathrm{Fe} / \mathrm{H}]^{\mathrm{CG}}$} & linear & -1.37 & 0.18 \\
& quadratic & -1.41 & 0.21 \\
{$[\mathrm{M} / \mathrm{H}]$} & linear & -1.18 & 0.19 \\
& quadratic & -1.22 & 0.20 \\
\hline
\end{tabular}

excluding the 4 most metal-poor stars in Fig. 8) are given in Table 7 for both scales, along with the results on the Zinn \& West (1984) scale for ease of comparison with previous literature. The metallicities of individual Leo I stars are listed in Table 3. Using the CG97 scale, the distribution is centred at $[\mathrm{Fe} / \mathrm{H}] \simeq-1.4$ with a standard deviation $\sigma_{[\mathrm{Fe} / \mathrm{H}]} \simeq 0.2$. For the $[\mathrm{M} / \mathrm{H}]$ calibration, the average is $[\mathrm{M} / \mathrm{H}] \simeq-1.2$ with the same scatter.

The results obtained from the linear and quadratic calibrations are very similar in all cases, as expected since most of the Leo I stars have metallicities lower than $[\mathrm{Fe} / \mathrm{H}]=-1.0$. The choice of the linear or quadratic relation only affects the metallicity of 4 metal-poor stars, having $[\mathrm{M} / \mathrm{H}] \lesssim-2$. In this range, the calibration is extrapolated beyond the most metal-poor globular cluster, which makes the metallicity of the 4 stars quite uncertain and dependent on the adopted calibration. In the case of the CG97 calibration, which yields the lowest extrapolated values, the 4 stars have $-2.6<[\mathrm{Fe} / \mathrm{H}]<-2.2$. Visual inspection of the targets on a Leo I image indicates normal star-like profiles (i.e. no blends). We therefore conclude that, while a few stars may have low metallicity, there is so far no evidence of extremely metal-poor stars in Leo I. Spectral synthesis methods (see, e.g., Kirby et al. 2008) will be used in a future paper to obtain more secure metallicity estimates for these metal-poor stars from a different spectral interval.

Our determination of the metallicity of Leo I agrees well with the results of Bosler et al. (2007) $([\mathrm{Fe} / \mathrm{H}]=-1.34)$ and Koch et al. (2007b) $([\mathrm{Fe} / \mathrm{H}]=-1.31)$, in particular when a linear calibration is used as in the previous papers. On the other hand, our $[\mathrm{M} / \mathrm{H}]$ values are in better agreement with the $[\mathrm{Ca} / \mathrm{H}]$ results of Bosler et al. (2007). Using 9 stars in common with Bosler et al. (2007) and 5 stars in common with Koch et al. (2007b), we compared the metallicities star-by-star. The mean differences are $\Delta[\mathrm{Fe} / \mathrm{H}]^{\mathrm{CG}}=-0.04 \pm 0.14$ (rms of the sample) and $\Delta[\mathrm{Fe} / \mathrm{H}]^{\mathrm{CG}}=-0.17 \pm 0.11$, respectively, in agreement with the shifts between the mean values of the MDFs. The rms values are consistent with our measurement error (see next section).

\subsection{Intrinsic metallicity dispersion and clues on the evolution of Leol}

The observed MDF in Fig. 8 is the convolution of the intrinsic metallicity distribution of stars in Leo I and the measurement errors. The real abundance spread can be estimated by adopting a Gaussian model for the intrinsic MDF, which yields $\sigma_{\text {OBS }}^{2}=\sigma_{0}^{2}+\sigma_{\text {err }}^{2}$, where $\sigma_{\text {OBS }}$ is the observed metallicity dispersion, $\sigma_{0}$ is the intrinsic dispersion, and $\sigma_{\text {err }}$ is the measurement error.

If we adopt a quadratic $[\mathrm{M} / \mathrm{H}]$ calibration and a typical measurement error $\sigma_{\text {err }} \simeq 0.14$ dex, the measurement scatter largely contributes to the observed metallicity dispersion. The observed scatter implies an intrinsic metallicity dispersion $\sigma_{[\mathrm{Fe} / \mathrm{H}], 0}=0.14$ for the CG97 scale and $\sigma_{[\mathrm{M} / \mathrm{H}], 0}=0.08$ for $[\mathrm{M} / \mathrm{H}]$. The intrinsic abundance dispersion of Leo I stars is therefore very small, even

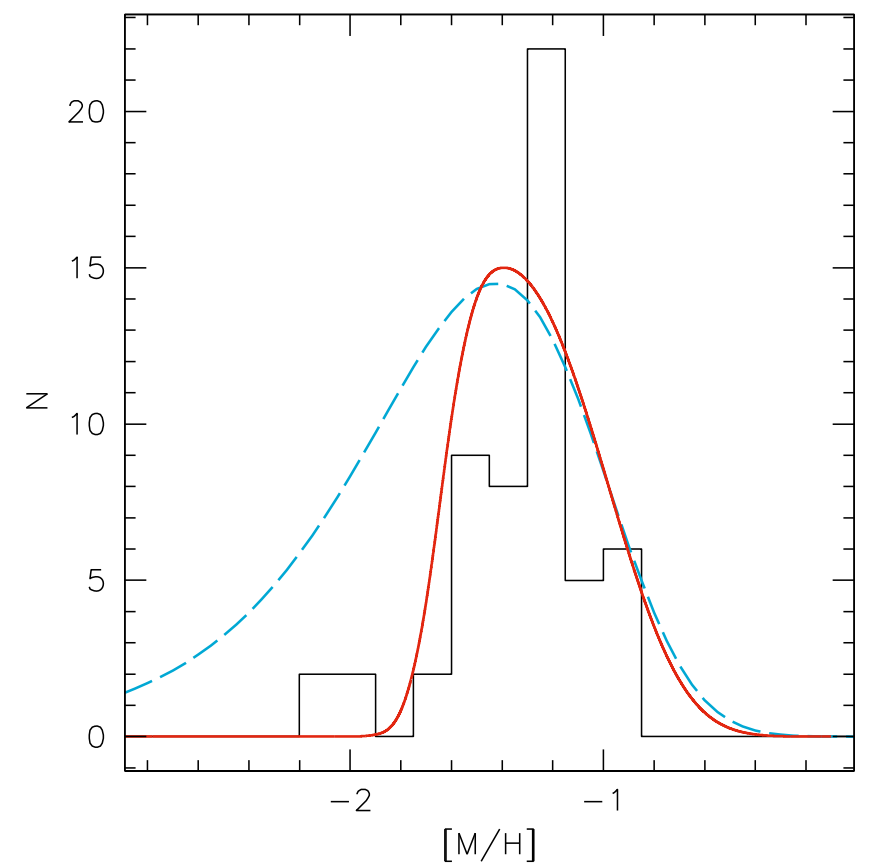

Fig. 9. The MDF of Leo I RGB stars on the $[\mathrm{M} / \mathrm{H}]$ metallicity scale, compared with a simple model with a low effective yield (dashed line) and a model with a prompt initial enrichment (solid line).

smaller than previously thought, and this happens in spite of the relatively wide range of ages of the stellar populations. This is an important constraint to the chemical evolution across the life of the galaxy.

In order to model the metallicity distribution and the chemical evolution of Leo I, detailed models have to be put forth (such as those of Lanfranchi \& Matteucci 2007) properly taking into account the chemical and dynamical evolution of the galaxy. However, some order-of-magnitude physical insight on the evolution of Leo I can already be obtained using basic considerations. The metallicity distribution of RGB stars in Leo I is compared in Fig. 9 with a simple closed-box model with a low effective yield consistent with a continuous loss of gas (e.g., Pagel 1997). In order to reproduce the peak of the observed $\mathrm{MDF}$, we have to adopt an effective yield $y=0.025 Z_{\odot}$, and $y=0.040 Z_{\odot}$, for the distributions based on the $[\mathrm{Fe} / \mathrm{H}]$ and $[\mathrm{M} / \mathrm{H}]$ metallicity scales, respectively. This is clearly much lower than the value found in the solar vicinity $\left(y=1.2 Z_{\odot}\right.$; e.g. Portinari et al. 2004), in a way consistent with the loss of metals driven by a galactic wind (Hartwick 1976; Pagel 1997). Still, even allowing for a gas outflow, the number of metal-poor stars is largely overestimated by the simple model, as shown in Fig. 9. The fit is considerably improved by assuming a prompt early enrichment with an initial metallicity $Z_{0}=0.02 Z_{\odot}([\mathrm{Fe} / \mathrm{H}]=$ -1.7) (continuous line in Fig. 9). Although very simplistic, this conclusion agrees with the finding that the metal-poor tail of the MDF in 4 Local Group dwarf spheroidal galaxies (Helmi et al. 2006) is significantly different from that of the Galactic halo, lacking stars below $[\mathrm{Fe} / \mathrm{H}]=-3$. What this "toy model" tells us is that the narrow MDF of the Leo I stars can be understood as a combination of fast enrichment from an initial generation of stars, and subsequent loss of metals through outflows. This situation is common among Local Group dwarfs, but the MDF of Leo I is the narrowest observed to date (cf. Tolstoy et al. 2001; Pont et al. 2004; Koch et al. 2006, 2007a). 


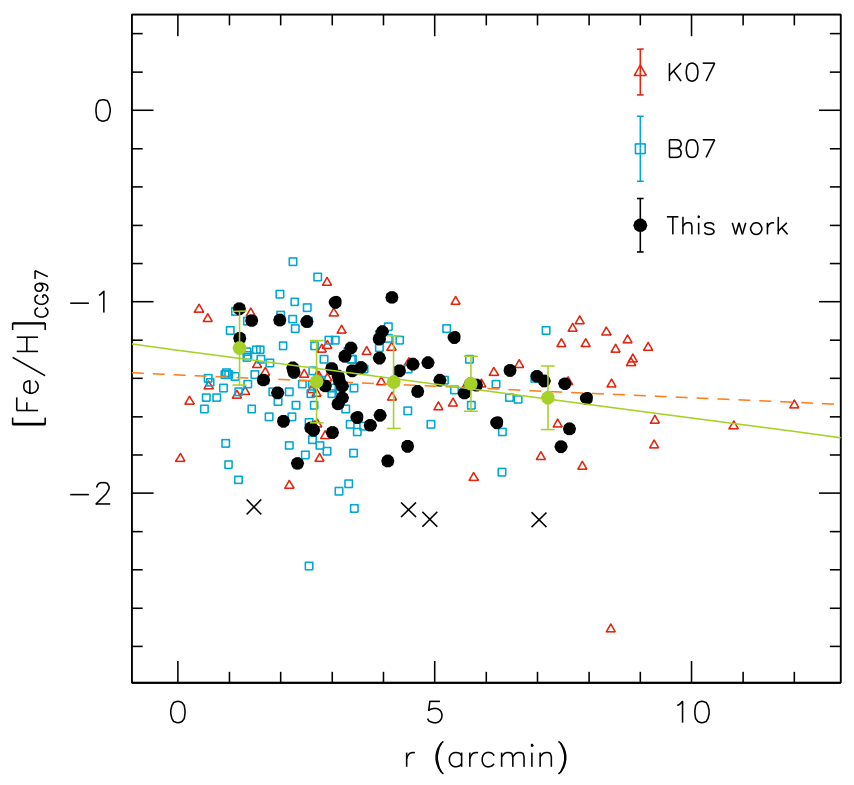

Fig. 10. Metallicities of Leo I stars on the $[\mathrm{Fe} / \mathrm{H}]$ scale of CG97, plotted against the elliptical radius (see text). Filled dots: data in this paper; open squares: data from Bosler et al. (2007); open triangles: Koch et al. (2007b); circles with error bars: our data, binned in 1.5 bins. The error bars of the binned data represent the abundance scatter $(1 \sigma)$ in each bin. The crosses are the 4 metal-poor stars in our sample. The typical errors of each study are shown in the upper right corner of the plot. The solid line is a fit to our (binned) data, while the dashed line represents a fit to all available spectroscopic data.

\subsection{Radial metallicity gradients}

Radial variations in the stellar populations are common in the dwarf spheroidals of the Local Group, where the younger and more metal-rich populations are often concentrated toward the galaxy centre (Harbeck et al. 2001; Saviane et al. 2001; Pont et al. 2004; Tolstoy et al. 2004; Koch et al. 2006). Leo I remains one of the few dSph's showing little evidence of a population gradient (Held et al. 2000; Koch et al. 2007b). In particular, the spectroscopic investigation of Koch et al. (2007b), extending to quite large radial distances, did not detect a significant metallicity gradient.

Our new spectroscopic sample of red giants allows us to further search for radial variations in the metallicity of Leo I stars. As the radial coordinate, we have adopted the semi-major axis $r$ of ellipses passing through the projected sky position of each star. The ellipses have the centre at $10^{\mathrm{h}} 08^{\mathrm{m}} 28^{\mathrm{s}} .1,+12^{\circ} 18^{\prime} 23^{\prime \prime}$ $(\mathrm{J} 2000)$ and a fixed position angle and ellipticity $\left(\mathrm{PA}=79^{\circ}\right.$, $\epsilon=0.21$; Irwin \& Hatzidimitriou 1995).

In Fig. 10, the metallicities of Leo I stars in our sample (see Table 3) are plotted against the distance from the centre. To directly compare our data with results from previous studies, we used the metallicity obtained from the calibration in terms of $[\mathrm{Fe} / \mathrm{H}]^{\mathrm{CG}}$. Data from Bosler et al. (2007) and Koch et al. (2007b) were shifted to account for the small differences in mean metallicity (of the order 0.1 dex or less) between the MDF's (Fig. 8). A linear relation was fitted both to our data alone (solid line) and to all available metallicity measurements in the literature (dashed line), excluding stars with $[\mathrm{Fe} / \mathrm{H}]<-2$.

The fit to our new FORS2 data yields a radial gradient of $-0.02 \mathrm{dex} \operatorname{arcmin}^{-1}$, or $-0.27 \mathrm{dex} \mathrm{Kpc}^{-1}$. In our sample, stars more metal-rich than $[\mathrm{Fe} / \mathrm{H}]=-1.3$ are only found in the central region of Leo I, with $a<5$.5. In contrast, the fit to the merged spectroscopic sample suggests a radially constant metallicity, in agreement with the conclusions of Koch et al. (2007b). To quantify the gradient, we have used a Kolmogorov-Smirnov test to compare the metallicity distributions of stars with $1.2<a<5^{\prime}$ (inner sample) and $5^{\prime}<a<8.2$ (outer sample), considering only the radial interval covered by our data. The two metallicity distributions are similar in shape, with the MDF in the inner region peaked at higher metallicity. The hypothesis that the inner and outer sample are drawn from the same parent population can be rejected at a $90 \%$ level using our data, and only at a nonsignificant $62 \%$ level using all spectroscopic data.

We conclude that, while our data provide a hint of a weak radial metallicity gradient in Leo I, the statistical significance of this result is at present low. More stars need to be observed, particularly in the outer region of the galaxy, before definite conclusions can be drawn.

\section{The age of Leol stars}

\subsection{The age-metallicity relation}

With the stellar metallicities of Leo I stars known from spectroscopy, ages could be estimated by comparing stars' locations in the CMD with a grid of theoretical isochrones (the models of Pietrinferni et al. 2004, were used to this purpose). Absolute magnitudes and dereddened colours were computed adopting a colour excess $E_{B-V}=0.04$, a total-to-differential extinction ratio $R_{V}=A_{V} / E_{B-V}=3.1$, and a true distance modulus $(m-M)_{0}=22.04$ (Held et al. 2009).

The stellar ages were interpolated in two steps. First, we used a set of theoretical isochrones of fixed age and different metallicities to find, for a star of given age and luminosity, a metallicity-colour relation and (from the known colour) an interpolated metallicity. This step was repeated for all model ages, yielding for each data point in the CMD a set of theoretical age-metallicity pairs each consistent with the star's magnitude and colour. This age-metallicity look-up table (spanning the full range from old, metal-poor stars to young, metal-rich stars) allowed us to compute an interpolated age for each star from its spectroscopic metallicity. We chose the $[\mathrm{M} / \mathrm{H}]$ scale for the input value, as the most directly related to the mass fraction of metals $(Z)$ used in stellar models. For a number of stars, ages could not be derived because the observed colour and/or magnitude were outside the range covered by the isochrones.

The method was checked against Galactic globular clusters with ages given by the literature. In particular, a small correction was applied to the isochrone colours so as to yield a correct age for NGC 5904 (M 5), assumed to be 12 Gyr (Sandquist et al. 1996) and the closest in metallicity to Leo I among the clusters listed in Table 5. Thus, our ages for Leo I stars are essentially referred to M 5, which is in our view the most correct approach given the considerable uncertainties in the isochrone colours.

The resulting ages are listed in Table 3 along with their uncertainties, estimated as follows. For each star, we performed a set of 100 Monte Carlo experiments by randomly varying the input quantities in intervals consistent with their uncertainties. We adopted a standard error $0.02 \mathrm{mag}$ in the $V$ magnitude, $0.05 \mathrm{mag}$ in $(B-V)$ colour, and $0.15 \mathrm{dex}$ in $[\mathrm{M} / \mathrm{H}]$. The latter was chosen conservatively large to account for the inherent uncertainties in the metallicity scale. The median and quartiles of the age distributions of randomly generated "stars" corresponding to each observed stars are listed in Table 3. In general, the uncertainties are of the order $50 \%$, which reasonably reflects the large uncertainties in the process. 


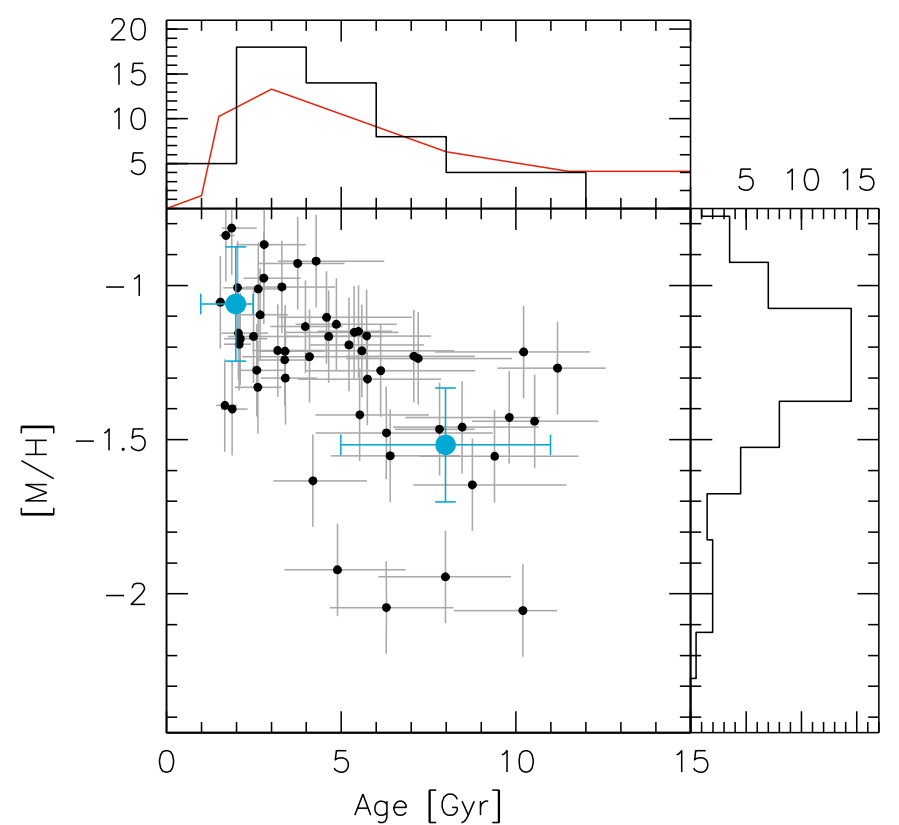

Fig. 11. The age-metallicity relation of Leo I RGB stars in our sample, on the $[\mathrm{M} / \mathrm{H}]$ scale. The error bars in age represent the first and third quartile of the confidence intervals obtained through Monte Carlo realisations (see text for details). For the metallicity errors the representative value discussed in Sect. 4.3 is adopted. Also shown are the metallicities from high-resolution spectroscopy of two Leo I RGB stars from Tolstoy et al. (2003) (big filled circles). The side histograms are the marginal distributions in metallicity and age. The solid line in the top panel represents the SFH derived by Dolphin (2002) from HST photometry, normalised to the total number of stars in our sample.

The age-metallicity relation derived from our data is shown in Fig. 11. The chemical evolution of Leo I seems to be very slow, in accord with the narrowness of the MDF. If the 4 metalpoor stars are excluded, there is a trend for stars younger than 5 Gyr to be on average more metal-rich by about $0.2-0.3$ dex. Similar conclusions were drawn, from a different data set, by Bosler et al. (2004). The scatter in the age-metallicity relation appears to be smaller than observed in other galaxies (Battaglia et al. 2006; Tolstoy et al. 2003, and references therein). Our data are in agreement with the results from high-resolution spectroscopy for 2 stars (Tolstoy et al. 2003).

We can use our age determinations also to obtain a SFH of Leo I. This can be done since our target selection was designed to avoid any bias in age and/or metallicity (see Sect. 2); the age distribution of our target stars is then proportional to the SFH. Our age measures are in agreement with the SFHs derived by HST photometry. In the upper panel of Fig. 11 our age distribution is compared with the Dolphin (2002) SFH, showing only small differences that can be explained by statistical fluctuations.

\subsection{Radial distribution of stellar ages}

To complete our analysis of the population gradients, we investigated the possible presence of a radial variation in the age of Leo I stars. Figure 12 shows the ages plotted against the elliptical distance. While old stars are found at all radii, young stars appear to be concentrated at small distances from the centre. To quantify this finding, we have plotted in Fig. 12 the cumulative distributions of two subsamples of stars in the inner $(r<3$ '9) and outer $(r>3$ '9) region, respectively. This limit was chosen to have the same number of stars in each subsample.

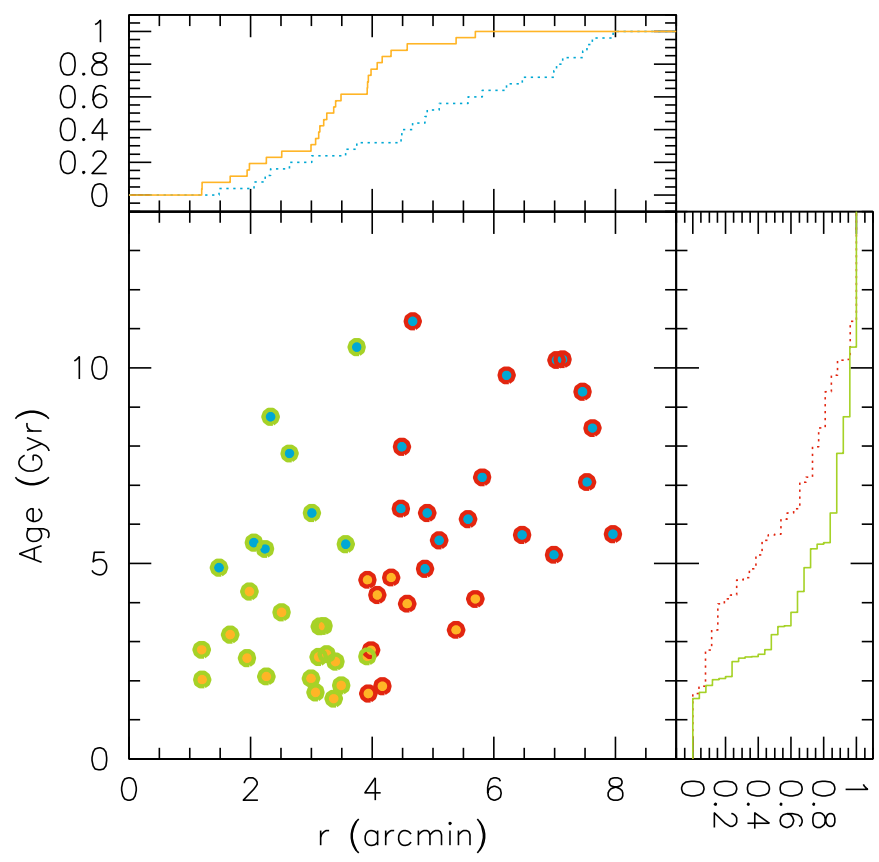

Fig. 12. Ages of the Leo I stars in our sample as a function of the elliptical radius (central panel). The upper panel shows the cumulative radial distributions of stars with ages smaller and larger (dotted line) than 4.6 Gyr. The cumulative age distributions of stars in the inner $(r<3.9)$ and outer $(r>3: 9$, dotted line) region are shown in the right panel (the different age and radial intervals are colour-coded in the electronic version of the journal). Younger stars appear to be more concentrated towards the centre of Leo I.

A Kolmogorov-Smirnov test indicates that the null hypothesis that the distributions are drawn from the same parent population can be rejected at $>99.9 \%$ confidence level. Similarly, we have plotted the cumulative distributions of two subsamples of stars with age $<4.6 \mathrm{Gyr}$ and $>4.6 \mathrm{Gyr}$. Also in this case, the probability of the null hypothesis can be rejected at a level $>99.9 \%$.

This suggestion of an age gradient among RGB stars is strengthened by the detection of a radial gradient in the fraction of upper-AGB stars in Leo I, which points to a concentration of intermediate-age populations towards the galaxy centre (Held et al. 2009).

\section{Summary and conclusions}

We have presented spectroscopic measurements of RGB stars in the LeoI dSph from observations carried out with the FORS2 spectrograph at the ESO VLT. We derived radial velocities for 57 stars with good $S / N$ ratio, 54 of which have been found to be Leo I members. Among these, 14 stars are in common with previous spectroscopic studies.

We measured the metallicities of RGB stars in Leo I from the equivalent widths of $\mathrm{Ca}$ II triplet lines, using the $[\mathrm{Fe} / \mathrm{H}]$ metallicity scales of Carretta \& Gratton (1997) and Zinn \& West (1984). In addition, we derived a new calibration tied to the $[\mathrm{M} / \mathrm{H}]$ ranking of Galactic globular clusters, which accounts for the abundance of both Fe-group and $\alpha$ elements.

The metallicity distribution (MDF) of Leo I stars is symmetric and very narrow. If we adopt a quadratic calibration of $\mathrm{Ca}$ II line strengths against $[\mathrm{Fe} / \mathrm{H}]$, the mean metallicity is $[\mathrm{Fe} / \mathrm{H}]=-1.41$ with a measured dispersion $0.21 \mathrm{dex}$ on the Carretta \& Gratton (1997) scale, in agreement with previous spectroscopic studies. The new $[\mathrm{M} / \mathrm{H}]$ calibration yields a mean 
value $[\mathrm{M} / \mathrm{H}]=-1.22$ with a dispersion 0.20 dex. By subtracting the measurement errors, we estimated a very low intrinsic metallicity dispersion, $\sigma_{[\mathrm{M} / \mathrm{H}]}=0.08$, which represents a constraint for modelling the chemical evolution of this isolated dwarf galaxy. As pointed out by previous studies, this narrow MDF is inconsistent with a simple "closed-box" chemical evolution model, even adopting a very low effective yield to account for galactic outflows expelling the metals produced by $\mathrm{SNe}$ winds. A prompt initial chemical enrichment may explain the very small number of extremely metal poor stars (we find only 4 stars with $[\mathrm{Fe} / \mathrm{H}]<-2$ ). Together, the two effects can explain the small abundance dispersion of Leo I stars, which gives the narrowest observed MDF among Local Group dwarf galaxies. However, detailed chemical evolution models (e.g., Lanfranchi \& Matteucci 2007) are needed to gain a complete picture of the evolution of Leo I.

Our data for RGB stars also provide an indication of a weak radial metallicity gradient in Leo I, of $-0.27 \mathrm{dex} \mathrm{Kpc}^{-1}$. In fact, all of our stars with $[\mathrm{M} / \mathrm{H}]>-1.3$ are found in the inner region $\left(r \lesssim 5^{\prime}\right)$. However, by combining our observations with previous spectroscopic datasets in the literature, the radial variation becomes insignificant. More observations in the outskirt of Leo I with a quality comparable to those presented here, are required to definitively establish the presence of an abundance gradient.

The metallicities of the RGB stars in our Leo I sample have been combined with existing photometric data to yield age estimates and an age-metallicity relation. Our age determinations are consistent with the SFH derived by Gallart et al. (1999) and Dolphin (2002) from HST photometry. The age-metallicity relation of Leo I red giants is quite flat, again suggesting a rapid initial enrichment. An increase in metal abundance by $\sim 0.2-0.3$ dex in the last 5 Gyr is possibly related to the main star-formation episode at intermediate ages. Since Leo I only hosts a minor old (>10 Gyr) stellar component, the chemical history of the galaxy is not well constrained at early epochs. Its most metal-poor stars must have formed out of a medium preenriched by a lost generation of stars, either before or after the galaxy had started assembling.

We have provided the first evidence of a radial variation in the ages of red giants in Leo I. Despite the uncertainties in age determination, our direct measurement of a radial variation of stellar ages seems quite convincing, with a Kormogorov-Smirnov test confirming, at a high level of statistical significance, that stars in the inner part of Leo I are on average younger than those in the outer regions. This result agrees with the conclusions of a parallel study of intermediateage AGB stars in Leo I from near-infrared photometry (Held et al. 2009). In the emerging scenario, the first generation of Leo I stars uniformly formed throughout this isolated dwarf spheroidal galaxy. The bulk of intermediate-age stars originated from an interstellar medium, poorly enriched by previous stellar generations mainly because of the effects of stellar winds. Younger stellar populations preferentially formed in the central regions, from gas somewhat enriched as seen from the agemetallicity relation in the last few Gyr. In this framework, our results on the radial distribution of Leo I stellar populations are not in contrast with previous results which found no gradients. The lack of detection of an age gradient by Held et al. (2000) can be explained considering that the mean age of the red-clump stars used by Held et al. (2000) as tracers of intermediate-age populations, is $\sim 5$ Gyr, which is older than that of upper AGB stars used by Held et al. (2009). As shown in Fig. 12, there are no clear radial variations in the age distribution of Leo I stars with ages greater than $\sim 5$ Gyr.

Acknowledgements. We thank A. Koch for providing us with unpublished data. M.G. wishes to thank the European Southern Observatory at Santiago, Chile for partial funding through DGDF and for hospitality during a visit in which this paper was partially written. This research was partially funded by PRIN MIUR 2007 "Galactic astroarchaeology: the local route to cosmology" (P.I. F. Matteucci).

\section{References}

Armandroff, T. E., \& Da Costa, G. S. 1991, AJ, 101, 1329

Armandroff, T. E., \& Zinn, R. 1988, AJ, 96, 92

Battaglia, G., Tolstoy, E., Helmi, A., et al. 2006, A\&A, 459, 423

Battaglia, G., Irwin, M., Tolstoy, E., et al. 2008, MNRAS, 383, 183

Bosler, T. L., Smecker-Hane, T. A., Cole, A., \& Stetson, P. B. 2004, in Origin and Evolution of the Elements, Carnegie Observatories Astrophysics Series, 4, ed. A. McWilliam, \& M. Rauch, Pasadena

Bosler, T. L., Smecker-Hane, T. A., \& Stetson, P. B. 2007, MNRAS, 378, 318 Carrera, R., Gallart, C., Pancino, E., \& Zinn, R. 2007, AJ, 134, 1298

Carretta, E., \& Gratton, R. G. 1997, A\&AS, 121, 95

Carretta, E., Cohen, J. G., Gratton, R. G., \& Behr, B. B. 2001, AJ, 122, 1469

Cole, A. A., Smecker-Hane, T. A., Tolstoy, E., Bosler, T. L., \& Gallagher, J. S. 2004, MNRAS, 347, 367

Cole, A. A., Tolstoy, E., Gallagher, III, J. S., \& Smecker-Hane, T. A. 2005, AJ, 129,1465

Da Costa, G. S., \& Armandroff, T. E. 1995, AJ, 109, 2533

Dolphin, A. E. 2002, MNRAS, 332, 91

Ferraro, F. R., Messineo, M., Fusi Pecci, F., et al. 1999, AJ, 118, 1738

Gallart, C., Freedman, W. L., Aparicio, A., Bertelli, G., \& Chiosi, C. 1999, AJ, 118,2245

Geisler, D., Smith, V. V., Wallerstein, G., Gonzalez, G., \& Charbonnel, C. 2005, AJ, 129, 1428

Gullieuszik, M., Held, E. V., Rizzi, L., et al. 2008, MNRAS, 388, 1185

Gullieuszik, M., Held, E. V., Rizzi, L., et al. 2007, A\&A, 467, 1025

Harbeck, D., Grebel, E. K., Holtzman, J., et al. 2001, AJ, 122, 3092

Hartwick, F. D. A. 1976, ApJ, 209, 418

Held, E. V., Clementini, G., Rizzi, L., et al. 2001, ApJ, 562, L39

Held, E. V., Gullieuszik, M., Rizzi, L., et al. 2009, MNRAS, submitted

Held, E. V., Saviane, I., Momany, Y., \& Carraro, G. 2000, ApJ, 530, L85

Helmi, A., Irwin, M. J., Tolstoy, E., et al. 2006, ApJ, 651, L121

Irwin, M., \& Hatzidimitriou, D. 1995, MNRAS, 277, 1354

Kirby, E. N., Guhathakurta, P., \& Sneden, C. 2008, ApJ, 682, 1217

Koch, A., Grebel, E. K., Wyse, R. F. G., et al. 2006, AJ, 131, 895

Koch, A., Grebel, E. K., Kleyna, J. T., et al. 2007a, AJ, 133, 270

Koch, A., Wilkinson, M. I., Kleyna, J. T., et al. 2007b, ApJ, 657, 241

Lanfranchi, G. A., \& Matteucci, F. 2007, A\&A, 468, 927

Mateo, M., Olszewski, E. W., \& Walker, M. G. 2008, ApJ, 675, 201

Pagel, B. E. J. 1997, Nucleosynthesis and Chemical Evolution of Galaxies, ed. B. E. J. Pagel (Cambridge, UK: Cambridge University Press), 392

Pietrinferni, A., Cassisi, S., Salaris, M., \& Castelli, F. 2004, ApJ, 612, 168

Pont, F., Zinn, R., Gallart, C., Hardy, E., \& Winnick, R. 2004, AJ, 127, 840

Portinari, L., Moretti, A., Chiosi, C., \& Sommer-Larsen, J. 2004, ApJ, 604, 579

Pritzl, B. J., Venn, K. A., \& Irwin, M. 2005, AJ, 130, 2140

Rutledge, G. A., Hesser, J. E., \& Stetson, P. B. 1997a, PASP, 109, 907

Rutledge, G. A., Hesser, J. E., Stetson, P. B., et al. 1997b, PASP, 109, 883

Salaris, M., Chieffi, A., \& Straniero, O. 1993, ApJ, 414, 580

Sandquist, E. L., Bolte, M., Stetson, P. B., \& Hesser, J. E. 1996, ApJ, 470, 910

Saviane, I., Held, E. V., Momany, Y., \& Rizzi, L. 2001, Mem. Soc. Astron. Ital., 72,773

Shetrone, M., Venn, K. A., Tolstoy, E., et al. 2003, AJ, 125, 684

Shetrone, M. D., Côté, P., \& Sargent, W. L. W. 2001, ApJ, 548, 592

Shetrone, M. D., Siegel, M. H., Cook, D. O., \& Bosler, T. 2009, AJ, 137, 62

Tolstoy, E., Irwin, M. J., Cole, A. A., et al. 2001, MNRAS, 327, 918

Tolstoy, E., Venn, K. A., Shetrone, M., et al. 2003, AJ, 125, 707

Tolstoy, E., Irwin, M. J., Helmi, A., et al. 2004, ApJ, 617, L119

van Dokkum, P. G. 2001, PASP, 113, 1420

Zinn, R., \& West, M. J. 1984, ApJS, 55, 45

Zoccali, M., Barbuy, B., Hill, V., et al. 2004, A\&A, 423, 507 